\title{
COMPANION LINEAR FUNCTIONALS AND SOBOLEV INNER PRODUCTS: A CASE STUDY*
}

\author{
ANTONIA M. DELGADO ${ }^{\dagger}$ AND FRANCISCO MARCELLÁN ${ }^{\ddagger}$
}

\begin{abstract}
The present paper deals with the solution of an inverse problem in the theory of orthogonal polynomials. It was motivated by a characterization result concerning sequences of polynomials orthogonal with respect to a Sobolev inner product when they can be recursively generated in terms of orthogonal polynomial sequences associated with the measure involved in the standard component. More precisely, we obtain the set of pairs of quasi-definite linear functionals such that their corresponding sequences of monic orthogonal polynomials $\left\{P_{n}\right\}$ and $\left\{R_{n}\right\}$ are related by a differential expression

$$
\frac{R_{n+1}^{\prime}(x)}{n+1}+b_{n} \frac{R_{n}^{\prime}(x)}{n}=P_{n}(x)+a_{n} P_{n-1}(x),
$$

where $b_{n} \neq 0$ for every $n \in \mathbb{N}$.
\end{abstract}

Key words. Semiclassical linear functionals, orthogonal polynomials, inverse problems, perturbations of linear functionals

AMS subject classifications. Primary 33C45, 42C05

1. Introduction. Consider a linear functional $\mathcal{U}$ in the linear space $\mathbb{P}$ of polynomials with real coefficients. $\mathbb{P}_{n}$ denotes the linear subspace of polynomials of degree at most $n$.

We introduce the sequence $\left\{u_{n}\right\}$ of moments associated with the linear functional $\mathcal{U}$, where $u_{n}=\left\langle\mathcal{U}, x^{n}\right\rangle, n \in \mathbb{N}$. The linear functional $\mathcal{U}$ is said to be quasi-definite [5] if the principal submatrices of the Hankel matrix $H=\left(u_{i+j}\right)_{i, j=0}^{\infty}$ are nonsingular. In such a situation there exists a sequence $\left\{R_{n}\right\}$ of monic polynomials such that

(i) $\operatorname{deg} R_{n}=n$,

(ii) $\left\langle\mathcal{U}, R_{n} R_{m}\right\rangle=k_{n} \delta_{n, m}, n, m \in \mathbb{N}$,

with $k_{n} \neq 0 . \quad\left\{R_{n}\right\}$ is said to be the sequence of monic polynomials orthogonal (SMOP) with respect to the linear functional $\mathcal{U}$. If the principal submatrices of $H$ are positive definite, then the linear functional $\mathcal{U}$ is said to be positive definite. In such a case, there exists a positive Borel measure $\mu$ supported in the real line such that $\langle\mathcal{U}, p\rangle=\int_{\mathbb{R}} p d \mu, p \in \mathbb{P}$.

It is very well known (see [5]) that $\left\{R_{n}\right\}$ is a SMOP with respect to a quasidefinite linear functional $\mathcal{U}$ if and only if there exist sequences of real numbers $\left\{\beta_{n}\right\}$ and $\left\{\gamma_{n}\right\}$ with $\gamma_{n} \neq 0$ for every $n \in \mathbb{N}$ such that

$$
x R_{n}(x)=R_{n+1}(x)+\beta_{n} R_{n}(x)+\gamma_{n} R_{n-1}(x) .
$$

This is the so-called three-term recurrence relation that plays a key role in the analysis of sequences of orthogonal polynomials.

In the last years an increasing attention was paid to inner products like

$$
\langle p, q\rangle=\int_{\mathbb{R}} p q d \mu_{0}+\lambda \int_{\mathbb{R}} p^{\prime} q^{\prime} d \mu_{1},
$$

\footnotetext{
*Received January 16, 2004; accepted for publication November 17, 2004.

${ }^{\dagger}$ Departamento de Matemáticas, Universidad Carlos III de Madrid, Avenida de la Universidad, 30. 28911 Leganés, Madrid, Spain (adelgado@math.uc3m.es).

${ }^{\ddagger}$ Departamento de Matemáticas, Universidad Carlos III de Madrid, Avenida de la Universidad, 30. 28911 Leganés, Madrid, Spain (pacomarc@ing.uc3m.es).
} 
where $\mu_{0}, \mu_{1}$ are positive Borel measures supported in the real line, $\lambda \in \mathbb{R}^{+}$, and $p, q \in \mathbb{P}$.

In such conditions, we can define a sequence of monic polynomials $\left\{Q_{n}(x ; \lambda)\right\}$ orthogonal with respect to $(1.2)$ such that

(i) $\operatorname{deg} Q_{n}(x ; \lambda)=n$,

(ii) $\left\langle Q_{n}(x ; \lambda), Q_{m}(x ; \lambda)\right\rangle=k_{n} \delta_{n, m}$ and $k_{n} \neq 0$.

In [6] the concept of coherent pairs of measures is introduced as follows.

Taking into account that the coefficients of $Q_{n}(x ; \lambda)$ are rational functions of $\lambda$ with numerator of degree less than or equal than the degree of the denominator, there exists a monic polynomial $W_{n+1}$ of degree $n+1$ such that

$$
W_{n+1}(x)=\lim _{\lambda \rightarrow \infty} Q_{n+1}(x ; \lambda) .
$$

It is straightforward to prove from (1.2) that

$$
\int_{\mathbb{R}} W_{n+1}^{\prime}(x) q^{\prime}(x) d \mu_{1}=0
$$

for every $q \in \mathbb{P}_{n}$. This means that $W_{n+1}^{\prime}(x)=(n+1) P_{n}(x)$, where $\left\{P_{n}\right\}$ denotes the sequence of monic polynomials orthogonal with respect to $\mu_{1}$, i.e. $\int_{\mathbb{R}} P_{n} P_{m} d \mu_{1}=$ $\delta_{n, m} k_{n}$ with $k_{n} \neq 0$. Furthermore

$$
\int_{\mathbb{R}} W_{n+1}(x) d \mu_{0}=0, \quad n \geq 0 .
$$

On the other hand, from the expansion

$$
P_{n}(x)=\sum_{k=1}^{n+1} b_{n, k} \frac{R_{k}^{\prime}(x)}{k}, n \geq 0,
$$

where $\left\{R_{n}\right\}$ is the SMOP with respect to $\mu_{0}$, we get

$$
\frac{W_{n+1}^{\prime}(x)}{n+1}=\sum_{k=1}^{n+1} b_{n, k} \frac{R_{k}^{\prime}(x)}{k}, n \geq 0,
$$

i.e.,

$$
\frac{W_{n+1}(x)}{n+1}=\sum_{k=1}^{n+1} b_{n, k} \frac{R_{k}(x)}{k}, n \geq 0,
$$

taking into account the constant of integration vanishes according to (1.3).

If in (1.4) $b_{n, k}=0$ for $1 \leq k \leq n-1$ and $b_{n}=b_{n, n} \neq 0$, then the pair of measures $\left(\mu_{0}, \mu_{1}\right)$ is said to be coherent [6].

On the other hand, from the Fourier expansion of the polynomial $W_{n+1}$ in terms of the polynomial sequence $Q_{n}(x ; \lambda)$ we get

$$
W_{n+1}(x)=Q_{n+1}(x ; \lambda)+\sum_{j=0}^{n} \beta_{n+1, j}(\lambda) Q_{j}(x ; \lambda), n \geq 0 .
$$


Assuming that $\left(\mu_{0}, \mu_{1}\right)$ is a coherent pair of measures we get for $0 \leq j \leq n-1$

$$
\begin{aligned}
\beta_{n+1, j}(\lambda) & =\frac{\left\langle W_{n+1}(x), Q_{j}(x ; \lambda)\right\rangle}{\left\langle Q_{j}(x ; \lambda), Q_{j}(x ; \lambda)\right\rangle} \\
& =\frac{\int_{\mathbb{R}} W_{n+1}(x) Q_{j}(x ; \lambda) d \mu_{0}+\lambda \int_{\mathbb{R}} W_{n+1}^{\prime}(x) Q_{j}^{\prime}(x ; \lambda) d \mu_{1},}{\left\langle Q_{j}(x ; \lambda), Q_{j}(x ; \lambda)\right\rangle} \\
& =0
\end{aligned}
$$

taking into account (1.5). Thus $W_{n+1}(x)=Q_{n+1}(x ; \lambda)+c_{n}(\lambda) Q_{n}(x ; \lambda)$, and together with (1.5) we get

$$
Q_{n+1}(x ; \lambda)+c_{n}(\lambda) Q_{n}(x ; \lambda)=R_{n+1}(x)+\frac{n+1}{n} b_{n} R_{n}(x), n \geq 1,
$$

where $c_{n}(\lambda) \neq 0$.

The relation (1.6) has been used as a basic tool for analysis of asymptotic properties of the polynomials $\left\{Q_{n}(x ; \lambda)\right\}$ (see for instance $[8,12,13,15]$ ).

In [14] the description of all coherent pairs of measures is done. As a conclusion, if $\left(\mu_{0}, \mu_{1}\right)$ is a pair of coherent measures, i.e., the corresponding sequences $\left\{R_{n}\right\}$ and $\left\{P_{n}\right\}$ of monic orthogonal polynomials are related by

$$
P_{n}(x)=\frac{R_{n+1}^{\prime}(x)}{n+1}+b_{n} \frac{R_{n}^{\prime}(x)}{n}, n \geq 1,
$$

then (1.6) holds. That the converse result is not true in general was proved in [7]. Indeed, if (1.6) holds, then

$$
\int_{\mathbb{R}}\left(\frac{R_{n+1}^{\prime}(x)}{n+1}+b_{n} \frac{R_{n}^{\prime}(x)}{n}\right) p^{\prime}(x) d \mu_{1}=0, n \geq 1,
$$

for every $p \in \mathbb{P}_{n-1}$. This means that

$$
\frac{R_{n+1}^{\prime}(x)}{n+1}+b_{n} \frac{R_{n}^{\prime}(x)}{n}=P_{n}(x)+a_{n} P_{n-1}(x), n \geq 1,
$$

with $b_{n} \neq 0$. Notice that we recover the coherent case if $a_{n}=0$ for every $n \in \mathbb{N}$.

Conversely, if (1.8) holds, then we have (1.7) for $p \in \mathbb{P}_{n-1}$. But, on the other hand, it is straightforward to prove

$$
\int_{\mathbb{R}}\left(\frac{R_{n+1}(x)}{n+1}+b_{n} \frac{R_{n}(x)}{n}\right) p(x) d \mu_{0}=0, n \geq 1 .
$$

Then, $\frac{R_{n+1}(x)}{n+1}+b_{n} \frac{R_{n}(x)}{n}$ is a monic polynomial orthogonal to $\mathbb{P}_{n-1}$ with respect to the Sobolev inner product. As a consequence, (1.6) follows.

Our work is focused in the analysis of the pair of linear functionals $(\mathcal{V}, \mathcal{U})$ such that the corresponding sequences of monic orthogonal polynomials $\left\{R_{n}\right\}$ and $\left\{P_{n}\right\}$, respectively, are related by (1.8).

If we write $T_{n}(x)=\frac{R_{n+1}^{\prime}(x)}{n+1}$, then relation (1.8) becomes

$$
T_{n}(x)+b_{n} T_{n-1}(x)=P_{n}(x)+a_{n} P_{n-1}(x), n \geq 0 .
$$

Notice that if $\mathcal{V}$ is a classical linear functional (Hermite, Laguerre, Jacobi and Bessel), then $\left\{T_{n}\right\}$ is again a sequence of classical orthogonal polynomials. This problem has 
been solved in [1] in the context of inverse problems for orthogonal polynomials. In [3] and [4] some particular examples of (1.6) when $\mathcal{V}$ is a polynomial perturbation of degree 1 of the Laguerre or Jacobi linear functional are studied. These remarkable examples prove that (1.6) holds despite the fact that $\left(\mu_{0}, \mu_{1}\right)$ is not a coherent pair. Some partial extensions of the above questions have been considered in [9], [10] as well as [11].

Applying the linear functional $\mathcal{U}$ to equation (1.9), we can compute $\left\langle\mathcal{U}, T_{n}\right\rangle$ in terms of $\left\langle\mathcal{U}, T_{n-1}\right\rangle$,

$$
\left\langle\mathcal{U}, T_{n}\right\rangle=-b_{n}\left\langle\mathcal{U}, T_{n-1}\right\rangle, n \geq 2 .
$$

On the other hand, if we assume that the linear functional $\mathcal{U}$ is normalized by $\langle\mathcal{U}, 1\rangle=$ 1 , then we easily get $\left\langle\mathcal{U}, T_{1}\right\rangle=a_{1}-b_{1}$. For technical reasons, we will assume $a_{1} \neq b_{1}$.

The structure of the paper is as follows. In Section 3 we characterize the pairs of linear functionals $(\mathcal{V}, \mathcal{U})$ such that $(1.8)$ holds. We prove that, under this assumption, one of them must be a semiclassical linear functional of class at most 1. In Section 4 we analyze the companion of $\mathcal{V}$ if we assume that $\mathcal{V}$ is semiclassical of class at most 1. We describe all of their companions taking into account that $\mathcal{V}$ is either classical or semiclassical of class 1 . In particular, we obtain coherent pairs which appear when $a_{n}=0$ for any $n \in \mathbb{N}$. In Section 5 we study the companion of $\mathcal{U}$ if we assume $\mathcal{U}$ semiclassical of class at most 1 . We describe all their companions when $\mathcal{U}$ is either classical or semiclassical of class 1 . We obtain coherent pairs when $a_{n}=0$ for every $n \in \mathbb{N}$.

As a conclusion, we deduce all the pairs of linear functionals such that (1.8) holds. The study of the sequences $\left\{Q_{n}(x ; \lambda)\right\}$, when they exist, will be the main aim of a future work.

2. Background. In the linear space $\mathbb{P}^{\prime}$ of linear functionals we can introduce some algebraic operations as follows [9].

Definition 2.1. Given $\mathcal{U} \in \mathbb{P}^{\prime}$ and $p \in \mathbb{P}$, the linear functional $p \mathcal{U}$ is defined by

$$
\langle p \mathcal{U}, q\rangle=\langle\mathcal{U}, p q\rangle, \quad q \in \mathbb{P} .
$$

This linear functional is said to be the left multiplication of a linear functional by a polynomial.

Notice that if $p(x)=\sum_{k=0}^{m} a_{k} x^{k}$, then the sequence of moments for the functional $p \mathcal{U}$ is given by

$$
\tilde{u}_{n}=\left\langle p \mathcal{U}, x^{n}\right\rangle=\sum_{k=0}^{m} a_{k} u_{k+n} .
$$

Definition 2.2. Given $\mathcal{U} \in \mathbb{P}^{\prime}$ and $p \in \mathbb{P}$, the linear functional $p^{-1} \mathcal{U}$ is defined by

$$
\left\langle p^{-1} \mathcal{U}, q\right\rangle=\left\langle\mathcal{U}, \frac{q(x)-L(x ; q)}{p(x)}\right\rangle, \quad q \in \mathbb{P},
$$

where $L(x ; q)$ denotes the interpolatory polynomial of $q$ in the zeros of $p$ taking into account the multiplicity. 
For instance, if $p(x)=x-a$, then

$$
\left\langle(x-a)^{-1} \mathcal{U}, q\right\rangle=\left\langle\mathcal{U}, \frac{q(x)-q(a)}{x-a}\right\rangle, \quad q \in \mathbb{P} .
$$

If $p(x)=(x-a)^{2}$, then

$$
\left\langle(x-a)^{-2} \mathcal{U}, q\right\rangle=\left\langle\mathcal{U}, \frac{q(x)-q(a)-q^{\prime}(a)(x-a)}{(x-a)^{2}}\right\rangle, \quad q \in \mathbb{P} .
$$

DEFINITION 2.3. Given a linear functional $\mathcal{U}$ we can introduce a new linear functional $\tilde{\mathcal{U}}$ such that

$$
\langle\tilde{\mathcal{U}}, p(x)\rangle=\langle\mathcal{U}, p(a x+b)\rangle, \quad p \in \mathbb{P} .
$$

This means that the moments of $\tilde{\mathcal{U}}$ are $\tilde{u}_{n}=\left\langle\mathcal{U},(a x+b)^{n}\right\rangle, n \in \mathbb{N}$.

Notice that if $\mathcal{U}$ is a quasi-definite linear functional and denote by $\left\{R_{n}\right\}$ the corresponding sequence of monic orthogonal polynomials, then $\tilde{\mathcal{U}}$ is a quasi-definite linear functional and the corresponding sequence $\left\{\tilde{R}_{n}\right\}$ of monic orthogonal polynomials is given by $\tilde{R}_{n}(x)=a^{n} R_{n}\left(\frac{x-b}{a}\right)$. Indeed

$$
\begin{aligned}
\left\langle\tilde{\mathcal{U}}, \tilde{R}_{n}(x) \tilde{R}_{m}(x)\right\rangle & =\left\langle\mathcal{U}, \tilde{R}_{n}(a x+b) \tilde{R}_{m}(a x+b)\right\rangle \\
& =\left\langle\mathcal{U}, a^{n} R_{n}(x) a^{m} R_{m}(x)\right\rangle \\
& =a^{n+m}\left\langle\mathcal{U}, R_{n}(x) R_{m}(x)\right\rangle \\
& =a^{n+m} k_{n} \delta_{n, m} .
\end{aligned}
$$

Definition 2.4. Given $a \in \mathbb{C}$, the Delta Dirac linear functional at a is defined as

$$
\langle\delta(x-a), p(x)\rangle=p(a)
$$

for every $p \in \mathbb{P}$.

Notice that the moments of this linear functional are $u_{n}=a^{n}$. As a straightforward consequence, the linear functional $\delta(x-a)$ is not quasi-definite.

Definition 2.5. Given $\mathcal{U} \in \mathbb{P}^{\prime}$, the derivative of this linear functional is a new linear functional DU such that

$$
\langle D \mathcal{U}, p(x)\rangle=-\left\langle\mathcal{U}, p^{\prime}(x)\right\rangle, \quad p \in \mathbb{P} .
$$

Notice that the moments of $D \mathcal{U}$ are $\tilde{u}_{n}=-n u_{n-1}, n \in \mathbb{N}$. As in the previous case, $D \mathcal{U}$ is not a quasi-definite linear functional taking into account $\tilde{u}_{0}=0$.

DEFINITION 2.6. Let $\left\{R_{n}\right\}$ be a sequence of monic polynomials with $\operatorname{deg} R_{n}=n$ for any $n \in \mathbb{N}$. A sequence of linear functionals $\left\{\alpha_{n}\right\}$ is said to be the dual basis of $\left\{R_{n}\right\}$ if $\left\langle\alpha_{n}, R_{m}\right\rangle=\delta_{n, m}$, for $n, m \in \mathbb{N}$.

In particular, if $\left\{R_{n}\right\}$ is a sequence of monic polynomials orthogonal with respect to a quasi-definite linear functional $\mathcal{V}$, then 
Proposition 2.7. The linear functional $\alpha_{n}$ can be explicitly expressed by

$$
\alpha_{n}=\frac{R_{n} \mathcal{V}}{\left\langle\mathcal{V}, R_{n}^{2}\right\rangle} .
$$

That is, $\left\langle\alpha_{n}, p\right\rangle=\left\langle\mathcal{V}, R_{n}^{2}\right\rangle^{-1}\left\langle\mathcal{V}, R_{n} p\right\rangle$ for any polynomial $p \in \mathbb{P}$.

LEMMA 2.8. Let $\left\{R_{n}\right\}$ be a sequence of monic polynomials orthogonal with respect to a linear functional $\mathcal{V}$, and denote by $T_{n}$ the monic derivative of $R_{n+1},(n+1) T_{n}=$ $R_{n+1}^{\prime}$ for $n \geq 0$. Then

$$
D \alpha_{n}^{\prime}=-(n+1) \alpha_{n+1}, n \geq 0,
$$

where $\left\{\alpha_{n}^{\prime}\right\}$ is the dual basis of the sequence of polynomials $\left\{T_{n}\right\}$.

Definition 2.9. A linear functional $\mathcal{U}$ is said to be classical if there exist two polynomials $\phi$ and $\psi$, with $\operatorname{deg} \phi \leq 2$ and $\operatorname{deg} \psi=1$, such that the following differential relation holds

$$
D(\phi \mathcal{U})=\psi \mathcal{U}
$$

This relation is called a distributional Pearson equation.

Taking into account the constraint about the degree of the polynomial $\phi$, if $\psi(x)=$ $a x+b$ then we can consider three situations.

1. $\operatorname{deg} \phi=0$. In such a case (2.2) becomes $D \mathcal{U}=\psi \mathcal{U}$ and thus the sequence of the moments $\left\{u_{n}\right\}$ satisfies $a u_{n+1}+b u_{n}+n u_{n-1}=0, n \geq 0$ (Hermite case).

2 . $\operatorname{deg} \phi=1$. In such a case we can use a change of variables such that $\phi(x)=$ $x$. Thus (2.2) becomes $D(x \mathcal{U})=\psi \mathcal{U}$ and the sequence of moments $\left\{u_{n}\right\}$ satisfies $a u_{n+1}+(b+n) u_{n}=0, n \geq 0$ (Laguerre case).

3. $\operatorname{deg} \phi=2$. In such a case three situations appear.

3.1. $\phi$ has a double zero. In such a case we can use a change of variables such that $\phi(x)=x^{2}$. Thus $(2.2)$ becomes $D\left(x^{2} \mathcal{U}\right)=\psi \mathcal{U}$, and the sequence of moments $\left\{u_{n}\right\}$ satisfies $(n+a) u_{n+1}+b u_{n}=0, n \geq 0$ (Bessel case).

3.2. $\phi$ has two simple and real zeros. In such a case we can consider a change of variable such that $\phi(x)=x^{2}-1$. Thus $(2.2)$ becomes $D\left(\left(x^{2}-1\right) \mathcal{U}\right)=\psi \mathcal{U}$, and the sequence of moments $\left\{u_{n}\right\}$ satisfies $(n+a) u_{n+1}+b u_{n}-n u_{n-1}=0, n \geq 0$ (Jacobi case).

3.3. $\phi$ has two conjugate complex zeros. In such a case we can consider a change of variable such that $\phi(x)=x^{2}+1$. Thus $(2.2)$ becomes $D\left(\left(x^{2}+1\right) \mathcal{U}\right)=\psi \mathcal{U}$, and the sequence of moments $\left\{u_{n}\right\}$ satisfies $(n+a) u_{n+1}+b u_{n}+n u_{n-1}=0, n \geq 0$ (Romanovski case). The Romanovski case will not be considered taking into account a complex linear change of variables reduces it to the Jacobi case.

On the other hand, the sequence $\left\{u_{n}\right\}$ is well-defined in cases 1 and 2 if $a \neq 0$ and in case 3 if $-a \notin \mathbb{N}$. This is the so-called admissibility condition in [9].

In order to the above linear functionals be quasi-definite we get

1. $\mathcal{U}=\mathcal{H}, \quad \psi(x)=-2 x$.

2. $\mathcal{U}=\mathcal{L}^{(\alpha)}, \quad \psi(x)=-x+\alpha+1,-\alpha \notin \mathbb{N}$.

3.1. $\mathcal{U}=\mathcal{B}^{(\alpha)}, \quad \psi(x)=(\alpha+2) x+2,-\alpha \notin \mathbb{N}$.

3.2. $\mathcal{U}=\mathcal{J}^{(\alpha, \beta)}, \quad \psi(x)=(\alpha+\beta+2) x-\beta+\alpha$,

$-\alpha \notin \mathbb{N},-\beta \notin \mathbb{N},-(\alpha+\beta) \notin \mathbb{N} \backslash\{1\}$. 
Definition 2.10. A linear functional is said to be positive definite when the principal submatrices of the Hankel matrix associated with its moments are positive definite.

In such a case, parameters $\gamma_{n}>0$ in the three-term recurrence relation (1.1), and we get an integral representation for such a linear functional

$$
\langle\mathcal{U}, p(x)\rangle=\int_{\mathbb{R}} p(x) d \mu(x),
$$

with $\mu$ a positive Borel measure supported in $\mathbb{R}$. In particular, in the classical cases:

1. $\langle\mathcal{H}, p(x)\rangle=\int_{\mathbb{R}} p(x) e^{-x^{2}} d x$.

2. If $\alpha>-1$, then $\left\langle\mathcal{L}^{(\alpha)}, p(x)\right\rangle=\int_{0}^{+\infty} p(x) x^{\alpha} e^{-x} d x$.

3.2. If $\alpha>-1, \beta>-1$, then $\left\langle\mathcal{J}^{(\alpha, \beta)}, p(x)\right\rangle=\int_{-1}^{1} p(x)(1-x)^{\alpha}(1+x)^{\beta} d x$.

Notice that the Bessel linear functional is an example of quasi-definite but not positive definite linear functional.

The sequences of monic polynomials orthogonal with respect to a quasi-definite classical linear functional can be characterized in several ways.

TheOREM 2.11 ([5, 9]). The following statements are equivalent,

(i) $\left\{R_{n}\right\}$ is a SMOP associated with a classical linear functional.

(ii) $\left\{\frac{R_{n+1}^{\prime}}{n+1}\right\}$ is a SMOP.

(iii) There exists a polynomial $\phi$ of degree at most two such that

$$
\phi(x) R_{n+1}^{\prime}(x)=r_{n} R_{n+2}(x)+s_{n} R_{n+1}(x)+t_{n} R_{n}(x),
$$

with $t_{n} \neq 0$.

As a natural extension, if in (2.2) we assume the only constraint $\operatorname{deg} \psi \geq 1$ and $\phi$ a polynomial, then the linear functional $\mathcal{U}$ is said to be semiclassical. A deep study of semiclassical linear functionals has been done in [9].

Notice that for a semiclassical linear functional there exists an infinite family of pairs of polynomials $(\phi, \psi)$ such that $(2.2)$ holds.

Definition 2.12. A semiclassical linear functional $\mathcal{U}$ is said of class $s$ when

$$
s=\min _{U}\{\max (\operatorname{deg} \phi-2, \operatorname{deg} \psi-1)\},
$$

where $U$ denotes the set of pairs of polynomials $(\phi, \psi)$ such that (2.2) holds.

In particular, classical orthogonal polynomials are semiclassical with class $s=0$.

THEOREM 2.13 ([9]). The following statements are equivalent,

(i) $\left\{R_{n}\right\}$ is a SMOP associated with a semiclassical linear functional of class $s$.

(ii) There exists a polynomial $\phi$ such that $\left\{\frac{R_{n+1}^{\prime}}{n+1}\right\}$ is a quasi-orthogonal family of monic polynomials of order $s$ with respect to the linear functional $\phi \mathcal{U}$, i.e.,

$$
\left\langle\phi \mathcal{U}, \frac{R_{n+1}^{\prime}}{n+1} p\right\rangle=0
$$

for every $p \in \mathbb{P}_{n-s-1}$. 
(iii) There exists a polynomial $\phi$ such that

$$
\phi(x) R_{n+1}^{\prime}(x)=\sum_{j=n-s}^{n+t} a_{n, j} R_{j}(x)
$$

with $a_{n, n-s} \neq 0$. Here $t=\operatorname{deg} \phi$.

A classification of semiclassical linear functionals of class $s=1$ is done in [2]. It will be very useful for our work.

3. Classification of the pairs $(\mathcal{U}, \mathcal{V})$. In this section we asume $\mathcal{V}, \mathcal{U}$ be two quasi-definite linear functionals such that the corresponding sequences of monic orthogonal polynomials, which we will denote by $\left\{R_{n}\right\}$ and $\left\{P_{n}\right\}$ respectively, satisfy the relation

$$
\frac{R_{n+1}^{\prime}(x)}{n+1}+b_{n} \frac{R_{n}^{\prime}(x)}{n}=P_{n}(x)+a_{n} P_{n-1}(x),
$$

with $b_{n} \neq 0$ for any $n \geq 1$. As in the previous section, we will denote by $T_{n}$ the monic derivatives of the polynomials $\left\{R_{n}\right\}$.

From now on we assume that $a_{1} \neq b_{1}$ and we study different relations between the linear functionals $\mathcal{U}$ and $\mathcal{V}$.

Lemma 3.1. Let $n \in \mathbb{N}$. Then there exists a monic polynomial $r_{n}$ of degree $n$ such that $\left\langle r_{n} \mathcal{U}, T_{m}\right\rangle=0$ for any $m \geq n+1$.

Proof. Let $r_{n}$ be a monic polynomial of degree $n$. Then, from relation (1.9) we get $\left\langle r_{n} \mathcal{U}, T_{m}\right\rangle=-b_{m}\left\langle r_{n} \mathcal{U}, T_{m-1}\right\rangle$ for every $m \geq n+2$. If we write

$$
r_{n}=P_{n}+\sum_{k=0}^{n-1} A_{n, k} P_{k},
$$

and using again (1.9), then we can consider the expansion of the polynomial $T_{n+1}$ with respect to $\left\{P_{n}\right\}$,

$$
T_{n+1}=P_{n+1}+\left(a_{n+1}-b_{n+1}\right) P_{n}+\ldots+(-1)^{n} b_{n+1} b_{n} \cdot \ldots \cdot b_{2}\left(a_{1}-b_{1}\right) P_{0} .
$$

Thus we compute the action of the linear functional $r_{n} \mathcal{U}$ over the polynomial $T_{n+1}$,

$$
\begin{aligned}
\left\langle r_{n} \mathcal{U}, T_{n+1}\right\rangle= & \left(a_{n+1}-b_{n+1}\right)\left\langle\mathcal{U}, P_{n}^{2}\right\rangle-b_{n-1}\left(a_{n}-b_{n}\right) A_{n, n-1}\left\langle\mathcal{U}, P_{n-1}^{2}\right\rangle \\
& +\ldots+(-1)^{n} b_{n+1} b_{n} \cdot \ldots \cdot b_{2}\left(a_{1}-b_{1}\right) A_{n, 0}\left\langle\mathcal{U}, P_{0}^{2}\right\rangle
\end{aligned}
$$

Finally, because of $a_{1} \neq b_{1}$, we can choose real numbers $A_{n, n-1}, \ldots, A_{n, 0}$ such that $\left\langle r_{n} \mathcal{U}, T_{n+1}\right\rangle=0$, and then the result follows. $\mathrm{Q}$

We observe that, a priori, we will eventually have some free parameters. In particular, we can choose $A_{n, n-1}=\ldots=A_{n, 1}=0$,

$$
A_{n, 0}=\frac{(-1)^{n+1}\left(a_{n+1}-b_{n+1}\right)\left\langle\mathcal{U}, P_{n}^{2}\right\rangle}{b_{n+1} b_{n} \cdot \ldots \cdot b_{2}\left(a_{1}-b_{1}\right)},
$$

and then we have $r_{n}=P_{n}+A_{n, 0}$.

As a consequence of the previous lemma, we prove the next result. 
Lemma 3.2. For any $n \in \mathbb{N}$, the linear functionals $\mathcal{U}$ and $\mathcal{V}$ are related by

$$
D\left[r_{n} \mathcal{U}\right]=-\varphi_{n+1} \mathcal{V}
$$

with $r_{n}$ a monic polynomial of degree $n$ and $\varphi_{n+1}$ a polynomial of degree at most $n+1$.

Proof. Let $r_{n}$ be the monic polynomial of degree $n$ introduced in Lemma 3.1. We consider the expansion of the linear functional $r_{n} \mathcal{U}$ in terms of the dual basis for the polynomials $\left\{T_{n}\right\}$,

$$
r_{n} \mathcal{U}=\sum_{k \geq 0} \lambda_{n, k} \alpha_{k}^{\prime}
$$

where $\lambda_{n, k}=\left\langle r_{n} \mathcal{U}, T_{k}\right\rangle$. From Lemma 3.1 these coefficients vanish for $k \geq n+1$, i.e.

$$
r_{n} \mathcal{U}=\sum_{k=0}^{n} \lambda_{n, k} \alpha_{k}^{\prime}
$$

Taking derivatives in the previous relation, Lemma 2.8 yields

$$
D\left[r_{n} \mathcal{U}\right]=-\sum_{k=0}^{n} \lambda_{n, k}(k+1) \alpha_{k+1}
$$

Finally, using the expression (2.1) for the linear functionals $\alpha_{k+1}$ we get the announced statement (3.2), with

$$
\varphi_{n+1}=\sum_{k=0}^{n} \frac{\lambda_{n, k}(k+1)}{\left\langle\mathcal{U}, R_{k+1}^{2}\right\rangle} R_{k+1}
$$

which is a polynomial of degree at most $n+1$.

For a sake of simplicity we will introduce the notation $\left\langle\mathcal{U}, R_{n}^{2}\right\rangle=\left\|R_{n}\right\|^{2}$.

The previous results will be particularly useful for $n=1,2$ as well as the explicit expressions for the polynomials $r_{1}, r_{2}, \varphi_{2}$, and $\varphi_{3}$. More precisely, for $n=1$ we have $r_{1}=P_{1}+A$ with

$$
A=\frac{\left(a_{2}-b_{2}\right)\left\|P_{1}\right\|^{2}}{b_{2}\left(a_{1}-b_{1}\right)}
$$

and,

$$
\varphi_{2}=\frac{2 a_{2}\left\|P_{1}\right\|^{2}}{b_{2}\left\|R_{2}\right\|^{2}} R_{2}+\frac{\left(a_{2}-b_{2}\right)\left\|P_{1}\right\|^{2}}{b_{2}\left(a_{1}-b_{1}\right)\left\|R_{1}\right\|^{2}} R_{1} .
$$

For $n=2, r_{2}=P_{2}+B P_{1}+C P_{0}$ with $B$ and $C$ verifying

$$
\left(a_{3}-b_{3}\right)\left\|P_{2}\right\|^{2}-b_{3}\left(a_{2}-b_{2}\right)\left\|P_{1}\right\|^{2} B+b_{2} b_{3}\left(a_{1}-b_{1}\right) C=0,
$$

and,

$$
\varphi_{3}=\frac{3 a_{3}\left\|P_{2}\right\|^{2}}{b_{3}\left\|R_{3}\right\|^{2}} R_{3}+\frac{2\left(B\left\|P_{1}\right\|^{2}+C\left(a_{1}-b_{1}\right)\right)}{\left\|R_{2}\right\|^{2}} R_{2}+\frac{C}{\left\|R_{1}\right\|^{2}} R_{1} .
$$

As a consequence of these lemmas, we get 
Corollary 3.3. In the previous conditions, there exist polynomials $\psi, \beta$, and $\alpha$, with $\operatorname{deg} \psi=2, \operatorname{deg} \beta \leq 4$, and $\operatorname{deg} \alpha \leq 3$ such that

$$
\begin{aligned}
& \psi(x) \mathcal{U}=\beta(x) \mathcal{V}, \\
& \alpha(x) \mathcal{U}=\beta(x) D \mathcal{U}, \\
& \psi(x) D \mathcal{U}=\alpha(x) \mathcal{V},
\end{aligned}
$$

where

$$
\begin{aligned}
& \psi=r_{1} r_{2}^{\prime}-r_{2}, \\
& \beta=r_{2} \varphi_{2}-r_{1} \varphi_{3}, \\
& \alpha=\varphi_{3}-r_{2}^{\prime} \varphi_{2} .
\end{aligned}
$$

Proof. From Lemma 3.2 with $n=1$ and $n=2$ we get

$$
\begin{aligned}
& \mathcal{U}+r_{1} D \mathcal{U}=-\varphi_{2} \mathcal{V}, \\
& r_{2}^{\prime} \mathcal{U}+r_{2} D \mathcal{U}=-\varphi_{3} \mathcal{V} .
\end{aligned}
$$

Then, by elimination of $D \mathcal{U}$ we obtain (3.4), by elimination of $\mathcal{V}$ we get (3.5), and, finally, by elimination of $\mathcal{U}$ we deduce (3.6).

4. $\mathcal{V}$ is semiclassical of class at most 1 . Since $\psi$ is a polynomial of degree 2 , we will give a classification for the linear functionals $\mathcal{U}, \mathcal{V}$ considering the different cases for the zeros of $\psi$. First, we deal with the case when $\psi$ has a double zero $\xi$.

4.1. Characterization of the pairs $(\mathcal{U}, \mathcal{V})$. In this section we study the case when the polynomial $\psi$ in Corollary 3.3 has a double zero. In fact, we prove that if (1.8) holds and $\psi$ has a double zero, then the linear functional $\mathcal{V}$ must be semiclassical of class at most 1 . Furthermore, we will characterize the linear functional $\mathcal{U}$ as a rational transformation of $\mathcal{V}$.

THEOREM 4.1. In the previous conditions, if the polynomial $\psi$ in (3.4) or (3.6) has a double zero $\xi \in \mathbb{R}$, then $\mathcal{V}$ is a semiclassical linear functional of class at most 1 , i.e. there exists $\tilde{\beta} \in \mathbb{P}$ of degree at most 3 , and $\varphi_{2}$ of degree al most 2 , such that

$$
D[\tilde{\beta} \mathcal{V}]=-\varphi_{2} \mathcal{V}
$$

Moreover,

$$
r_{1} \mathcal{U}=\tilde{\beta} \mathcal{V}
$$

Proof. If we take derivatives in (3.7) then $\psi^{\prime}=2 r_{1}$. Since $\xi$ is a double zero of $\psi$, we deduce that $\xi$ is also a zero of $r_{1}$, that is, $r_{1}(x)=x-\xi$.

Then, from (3.7) we get $r_{2}(\xi)=0$. Thus we can write $r_{2}=r_{1} s_{1}$, with $s_{1}$ a monic polynomial of degree 1 .

On the other hand, since $\beta(\xi)=r_{2}(\xi) \varphi_{2}(\xi)-r_{1}(\xi) \varphi_{3}(\xi)=0$, then $\beta=r_{1} \tilde{\beta}$, where $\tilde{\beta}=s_{1} \varphi_{2}-\varphi_{3}$ is a polynomial of degree at most 3 .

In such a situation, (3.8) and (3.9) become

$$
\begin{aligned}
& s_{1} \mathcal{U}+r_{2} D \mathcal{U}=-s_{1} \varphi_{2} \mathcal{V}, \\
& \left(r_{1}+s_{1}\right) \mathcal{U}+r_{2} D \mathcal{U}=-\varphi_{3} \mathcal{V},
\end{aligned}
$$


from where we obtain in a direct way (4.2).

In order to prove (4.1), we take derivatives in (4.2) and use (3.2) with $n=1$,

$$
D[\tilde{\beta} \mathcal{V}]=D\left[r_{1} \mathcal{U}\right]=-\varphi_{2} \mathcal{V} .
$$

Finally, since $\operatorname{deg} \tilde{\beta} \leq 3$ and $\operatorname{deg} \varphi_{2} \leq 2$, then $\mathcal{V}$ is a semiclassical linear functional of class at most $1 . \square$

The previous theorem allows us to give a complete classification of the pairs of linear functionals in this situation. To do that, we will discuss the cases where the relation (4.1) can be reduced to a classical condition.

From (4.1), we get

$$
\tilde{\beta} D \mathcal{V}=-\left(\varphi_{2}+\tilde{\beta}^{\prime}\right) \mathcal{V}
$$

Then, we must study the cases where the polynomials $\tilde{\beta}$ and $\varphi_{2}+\tilde{\beta}^{\prime}$ have at least one common zero as well as we assume that (4.3) can be simplified without the addition of Dirac masses in the resulting relations.

First, we will see how a linear change of variable affects a relation of Pearson-type for a linear functional.

Lemma 4.2. Let $\mathcal{U}$ be a linear functional satisfying the Pearson equation

$$
D[\phi(x) \mathcal{U}]=\psi(x) \mathcal{U} .
$$

Consider the linear functional $\hat{\mathcal{U}}$ defined from $\mathcal{U}$ by a linear change of variable as follows

$$
\langle\hat{\mathcal{U}}, p(x)\rangle=\left\langle\mathcal{U}, p\left(\frac{x-b}{a}\right)\right\rangle, p \in \mathbb{P} .
$$

Then, the linear functional $\hat{\mathcal{U}}$ satisfies

$$
D\left[\frac{1}{a^{t}} \phi(a x+b) \hat{\mathcal{U}}\right]=\frac{1}{a^{t-1}} \psi(a x+b) \hat{\mathcal{U}},
$$

where $t=\operatorname{deg} \phi$.

Next, we introduce some notation. We recall the expressions for the polynomials $\varphi_{2}$ and $\tilde{\beta}$,

$$
\begin{aligned}
& \varphi_{2}=\frac{2 a_{2}\left\|P_{1}\right\|^{2}}{b_{2}\left\|R_{2}\right\|^{2}} R_{2}+\frac{\left(a_{2}-b_{2}\right)\left\|P_{1}\right\|^{2}}{b_{2}\left(a_{1}-b_{1}\right)\left\|R_{1}\right\|^{2}} R_{1}, \\
& \tilde{\beta}=s_{1} \varphi_{2}-\varphi_{3}
\end{aligned}
$$

where $s_{1}(x)=x-c$ is a monic polynomial of degree 1 . If we make a linear change of variable $y \equiv a x+b$, for convenient values of $a$ and $b$ in each particular case, then we will denote by $\hat{\varphi}_{2}(x)=A^{-1} a^{1-t} \varphi_{2}(a x+b)$, and $\hat{\beta}(x)=A^{-1} a^{-t} \tilde{\beta}(a x+b) / a$ the corresponding transformed functions, where $t=\operatorname{deg} \tilde{\beta}$ and $A$ is the leading coefficient of $\tilde{\beta}$. We write

$$
\hat{\varphi}_{2}(x)=\eta_{2} x^{2}+\eta_{1} x+\eta_{0},
$$

with $\left|\eta_{2}\right|+\left|\eta_{1}\right| \neq 0$. Taking into account Lemma 4.2, up to this change of variable, relation (4.1) becomes $D[\hat{\beta} \mathcal{V}]=-\hat{\varphi}_{2} \mathcal{V}$. 
Keeping in mind this notation, we will analyze the possible classical cases in terms of the zeros of the polynomial $\tilde{\beta}$.

JACOBI AND BESSEL CASES: $\operatorname{deg} \tilde{\beta}=3$.

With the help of Lemma 4.2 all the possible cases for $\tilde{\beta}$ can be reduced, by a suitable linear change of variables, to the following three canonical situations:

$$
\begin{aligned}
& \tilde{\beta}(x)=x^{3}, \\
& \tilde{\beta}(x)=x^{2}(x-1), \\
& \tilde{\beta}(x)=x(x-1)(x-\lambda) .
\end{aligned}
$$

Then, we may assume that $\tilde{\beta}$ has this expression without loss of generality.

THEOREM 4.3. If $\tilde{\beta}(x)=x^{3}$, thus $\mathcal{V}$ is a classical linear functional if and only if $\eta_{0}=0$ and $\left(\eta_{2}+1\right) \beta_{0}^{\mathcal{V}}+\eta_{1}=0$, where $\beta_{0}^{\mathcal{V}}=-R_{1}(0)$. Then, $\mathcal{V}$ is the classical Bessel linear functional $\mathcal{B}^{(\alpha)}$ satisfying

$$
D\left[x^{2} \mathcal{V}\right]=-\left[\left(\eta_{2}+1\right) x+\eta_{1}\right] \mathcal{V}
$$

under the admissibility conditions $\eta_{1}=-2$ and $\eta_{2}+3 \notin \mathbb{N}$. Moreover, the linear functional $\mathcal{U}$ is given by

$$
\mathcal{U}=\left(x-\xi_{0}\right)^{-1} \mathcal{B}^{(\alpha+3)}+M \delta\left(x-\xi_{0}\right) .
$$

Proof. $\left[\hat{\varphi}_{2}+\hat{\beta}^{\prime}\right](0)=0$ if and only if $\hat{\varphi}_{2}(0)=\eta_{0}=0$. This gives us the first condition. Then $\hat{\varphi}_{2}+\hat{\beta}^{\prime}=x \phi_{1}(x)$, where

$$
\phi_{1}(x)=\left(\eta_{2}+3\right) x+\eta_{1} .
$$

Thus, we can simplify in (4.3) and obtain $x^{2} D \mathcal{V}=-\phi_{1}(x) \mathcal{V}+M \delta(x)$. In such conditions, the linear functional $\mathcal{V}$ is classical if and only if $M=0$. Previous expression applied to 1 yields $M=0$ if and only if

$$
\left\langle x^{2} D \mathcal{V}, 1\right\rangle+\left\langle\phi_{1}(x) \mathcal{V}, 1\right\rangle=0
$$

that is, $\left\langle\mathcal{V}, \phi_{1}(x)-2 x\right\rangle=0$. Finally, we get the second condition, $\left(\eta_{2}+1\right) \beta_{0}^{\mathcal{V}}+\eta_{1}=0$. The last statement is a direct consequence of (4.2).

TheOREm 4.4. If $\tilde{\beta}(x)=x^{2}(x-1)$ then, $\mathcal{V}$ is a classical linear functional if and only if one of the following situations appears.

i) $\eta_{0}=0$ and $\left(\eta_{2}+1\right) \beta_{0}^{\mathcal{V}}+\eta_{1}-1=0$. In this case, $\mathcal{V}$ is the translate of the classical Jacobi linear functional to the interval [0,1], satisfying

$$
D[x(x-1) \mathcal{V}]=-\left[\left(\eta_{2}+1\right) x+\eta_{1}-1\right] \mathcal{V},
$$

under the admissibility conditions $-\eta_{1}-\eta_{2}+1 \notin \mathbb{N}, \eta_{1} \notin \mathbb{N}$, and $-\eta_{2}+1 \notin \mathbb{N}$. Moreover, if the linear functional $\mathcal{V}=\mathcal{J}^{(\alpha, \beta)}$ is the Jacobi linear functional associated with the measure $d \mu_{0}=(1-x)^{\alpha}(1+x)^{\beta} d x$, then the linear functional $\mathcal{U}$ satisfies

$$
\mathcal{U}=(x-\xi)^{-1} \mathcal{J}^{(\alpha+1, \beta+2)}+M \delta(x-\xi),
$$

with $|\xi| \geq 1$. 
ii) $\eta_{2}+\eta_{1}+\eta_{0}+1=0$ and $\left(\eta_{2}+1\right) \beta_{0}^{\mathcal{V}}-\eta_{0}=0$. In this case, $\mathcal{V}=\mathcal{B}^{(\alpha)}$ is the classical Bessel linear functional, satisfying

$$
D\left[x^{2} \mathcal{V}\right]=-\left[\left(\eta_{2}+1\right) x-\eta_{0}\right] \mathcal{V}
$$

under the admissibility conditions $\eta_{0}=2$ and $\eta_{2}-3 \notin \mathbb{N}$. Thus, the linear functional $\mathcal{U}$ is

$$
\mathcal{U}=(x-\xi)^{-1}(x-1) \mathcal{B}^{(\alpha+2)}+M \delta(x-\xi) .
$$

Proof. In a similar way than in the previous theorem, we get condition i) from the case $\left[\hat{\varphi}_{2}+\hat{\beta}^{\prime}\right](0)=0$ and condition ii) from $\left[\hat{\varphi}_{2}+\hat{\beta}^{\prime}\right](1)=0 . \square$

THEOREM 4.5. If $\hat{\beta}(x)=x(x-1)(x-\lambda)$, then $\mathcal{V}$ is a classical linear functional if and only if one of the following situations holds.

i) $\eta_{0}+\lambda=0$ and $\left(\eta_{2}+1\right) \beta_{0}^{\mathcal{V}}+\eta_{1}-\lambda-1=0$. Then, $\mathcal{V}$ is the classical Jacobi linear functional translated to the interval $[\lambda, 1]$ or on $[1, \lambda]$, satisfying

$$
D[(x-1)(x-\lambda) \mathcal{V}]=-\left[\left(\eta_{2}+1\right) x+\eta_{1}-\lambda-1\right] \mathcal{V},
$$

under the admissibility conditions $\left(\eta_{1}-\lambda\left(1-\eta_{2}\right)\right) /(1-\lambda) \notin \mathbb{N},\left(1-\eta_{1}-\eta_{2}\right) /(1-\lambda) \notin \mathbb{N}$, and $1-\eta_{2} \notin \mathbb{N}$.

ii) $\eta_{2}+\eta_{1}+\eta_{0}-\lambda+1=0$ and $\left(\eta_{2}+1\right) \beta_{0}^{\mathcal{V}}-\eta_{0}=0$. Then, $\mathcal{V}$ is the Jacobi linear functional supported either on the interval $[0, \lambda]$ or $[\lambda, 0]$ and satisfying

$$
D[x(x-\lambda) \mathcal{V}]=-\left[\left(\eta_{2}+1\right) x-\eta_{0}\right] \mathcal{V}
$$

under the admissibility conditions $\eta_{0} / \lambda-\eta_{2} \notin \mathbb{N}, 1-\eta_{0} / \lambda \notin \mathbb{N}$, and $1-\eta_{2} \notin \mathbb{N}$.

iii) $\left(\eta_{2}+1\right) \lambda+\left(\eta_{1}-1\right) \lambda+\eta_{0}=0$ and $\left(\eta_{2}+1\right) \beta_{0}^{\mathcal{V}}-\eta_{0} / \lambda=0$. In such a case, $\mathcal{V}$ is the Jacobi linear functional on the interval $[0,1]$ satisfying

$$
D[x(x-1) \mathcal{V}]=\left[-\left(\eta_{2}+1\right) x+\frac{\eta_{0}}{\lambda}\right] \mathcal{V},
$$

under the admissibility conditions $\eta_{0} / \lambda-\eta_{2} \notin \mathbb{N}, 1-\eta_{0} / \lambda \notin \mathbb{N}$, and $1-\eta_{2} \notin \mathbb{N}$.

Moreover, in any of the three previous situations, if $\mathcal{V}=\mathcal{J}^{(\alpha, \beta)}$ is the Jacobi linear functional associated with the measure $d \mu_{0}=(1-x)^{\alpha}(1+x)^{\beta} d x$, then the linear functional $\mathcal{U}$ is given by

$$
\mathcal{U}=\left(x-\xi_{1}\right)^{-1}\left(x-\xi_{0}\right) \mathcal{J}^{(\alpha+1, \beta+1)}+M \delta\left(x-\xi_{1}\right),
$$

with either $\left|\xi_{0}\right|,\left|\xi_{1}\right| \geq 1$, or $\xi_{0}=\xi_{1}$ and $\left|\xi_{0}\right| \geq 1$.

Proof. In a similar way than in the previous theorem, we get condition i) from the case $\left[\hat{\varphi}_{2}+\hat{\beta}^{\prime}\right](0)=0$, condition ii) from $\left[\hat{\varphi}_{2}+\hat{\beta}^{\prime}\right](1)=0$, and condition iii) from $\left[\hat{\varphi}_{2}+\hat{\beta}^{\prime}\right](\lambda)=0$.

Notice that in every case with $\operatorname{deg} \tilde{\beta}=3$, the admissibility conditions yield $\eta_{2} \neq 0$. This means that the degree of the polynomial $\varphi_{2}$ in relation (4.1) must be 2 .

Proceeding in the same way as above, we analyze the cases when $\operatorname{deg} \tilde{\beta}=2$ and $\operatorname{deg} \tilde{\beta}=1$.

LAGUERRE CASES: $\operatorname{deg} \tilde{\beta}=2$.

If $\tilde{\beta}$ is of degree 2 then, by a linear change of variables we can make 


$$
\begin{aligned}
& \tilde{\beta}(x)=x^{2}, \\
& \tilde{\beta}(x)=x(x-1) .
\end{aligned}
$$

Hence, we may assume that $\tilde{\beta}$ has this expression without loss of generality.

Theorem 4.6. If $\tilde{\beta}(x)=x^{2}$, then (4.1) reduces to

$$
D[x \mathcal{V}]=-\left[\eta_{2} x+\eta_{1}+1\right] \mathcal{V}
$$

if and only if $\eta_{0}=0$ and $\eta_{2} \beta_{0}^{\mathcal{V}}+\eta_{1}=0$. As a consequence, $\mathcal{V}$ is the classical Laguerre functional under the admissibility conditions $\eta_{2}=1$ and $\eta_{1}+2 \notin \mathbb{N}$. Moreover, if $\mathcal{V}=$ $\mathcal{L}^{(\alpha)}$ is the Laguerre linear functional associated with the measure $d \mu_{0}=x^{\alpha} e^{-x} d x$, then the linear functional $\mathcal{U}$ satisfies

$$
\mathcal{U}=\left(x-\xi_{0}\right)^{-1} \mathcal{L}^{(\alpha+2)}+M \delta\left(x-\xi_{0}\right),
$$

with $\xi_{0} \leq 0$.

THEOREM 4.7. If $\tilde{\beta}(x)=x(x-1)$, thus $\mathcal{V}$ is a classical linear functional if and only if one of the following situations holds.

i) $\eta_{0}=1$ and $\eta_{2} \beta_{0}^{\mathcal{V}}+\eta_{1}+1=0$. In this case, $\mathcal{V}$ is the translated of the classical Laguerre functional satisfying the Pearson equation

$$
D[(x-1) \mathcal{V}]=-\left(\eta_{2} x+\eta_{1}+1\right) \mathcal{V},
$$

under the admissibility conditions $\eta_{2}=1$ and $\eta_{1}+3 \notin \mathbb{N}$.

ii) $\eta_{2}+\eta_{1}+\eta_{0}+1=0$ and $\eta_{2} \beta_{0}^{\mathcal{V}}-\eta_{0}=0$. Then, $\mathcal{V}$ is the classical Laguerre functional verifying the Pearson equation

$$
D[x \mathcal{V}]=-\left(\eta_{2} x-\eta_{0}\right) \mathcal{V},
$$

under the admissibility conditions $\eta_{2}=1$ and $1-\eta_{0} \notin \mathbb{N}$.

Moreover, if $\mathcal{V}=\mathcal{L}^{(\alpha)}$ is the Laguerre linear functional associated with the measure $d \mu_{0}=x^{\alpha} e^{-x} d x$, then the linear functional $\mathcal{U}$ satisfies

$$
\mathcal{U}=\left(x-\xi_{1}\right)^{-1}\left(x-\xi_{0}\right) \mathcal{L}^{(\alpha+1)}+M \delta\left(x-\xi_{1}\right),
$$

with either $\xi_{0}, \xi_{1} \leq 0$ or $\xi_{0}=\xi_{1}>0$.

HeRMite CASE: $\operatorname{deg} \tilde{\beta}=1$.

THEOREM 4.8. If $\operatorname{deg} \tilde{\beta}=1$ then, with an appropriate linear change of variable, we have $\hat{\beta}(x)=x$. In that situation, $\mathcal{V}$ is a classical linear functional if and only if $\eta_{0}+1=0$ and $\eta_{2} \beta_{0}^{\mathcal{V}}+\eta_{1}=0$. Thus, $\mathcal{V}$ is the translated of the classical Hermite linear functional verifying the Pearson equation

$$
D[\mathcal{V}]=-\left(\eta_{2} x+\eta_{1}\right) \mathcal{V}
$$

under the admissibility conditions $\eta_{2}=2$ and $\eta_{1}=1$. Moreover, if $\mathcal{V}=\mathcal{H}$ is the Hermite linear functional associated with the measure $d \mu_{0}=e^{-x^{2}} d x$, then the linear functional $\mathcal{U}$ satisfies

$$
\mathcal{U}=\mathcal{H}+M \delta(x) .
$$


Notice that in this case, both linear functionals $\mathcal{U}$ and $\mathcal{V}$ are symmetric, and then for $n=1$ relation (1.8) becomes,

$$
P_{1}+a_{1}=\frac{R_{2}^{\prime}}{2}+b_{1}
$$

This relation means $a_{1}=b_{1}$, a contradiction with our hypothesis. Then, the case stated in Theorem 4.8 is not possible.

4.2. Classification. Taking into account the results obtained in Section 4.1 we give a description of the linear functional $\mathcal{U}$ according to the different possibilities for $\mathcal{V}$ to be a classical or a semiclassical linear functional of class 1.

From Theorem 4.1, we have the following pairs of linear functionals when the polynomial $\psi$ in Corollary 3.3 has a double zero.

1. If $\operatorname{deg} \tilde{\beta} \leq 2$ and $\operatorname{deg} \varphi_{2}=1$, then the linear functional $\mathcal{V}$ associated with the measure $d \mu_{0}$ is a classical one, and we have the following possibilities for the measure $d \mu_{1}$ associated with $\mathcal{U}$,

$$
\begin{aligned}
d \mu_{0} & =(1-x)^{\alpha}(1+x)^{\beta} d x, \\
\left(J_{1,1}\right) \quad d \mu_{1} & =\frac{1}{x-\xi}(1-x)^{\alpha+1}(1+x)^{\beta+1} d x+M \delta(x-\xi),
\end{aligned}
$$

with $\alpha, \beta>-1$ and $|\xi| \geq 1$.

$\left(L_{1,1}\right) \quad d \mu_{0}=x^{\alpha} e^{-x} d x, \quad d \mu_{1}=\frac{1}{x-\xi} x^{\alpha+1} e^{-x} d x+M \delta(x-\xi)$,

with $\alpha>-1$ and $\xi \leq 0$.

These cases correspond to the positive-definite coherent pairs described in [14]. They satisfy relation (1.8) with $a_{n}=0$ for any $n \in \mathbb{N}$.

If $\mathcal{V}=\mathcal{B}^{(\alpha)}$ is the Bessel linear functional, then we have

$\left(B_{1,1}\right) \quad \mathcal{U}=(x-\xi)^{-1} \mathcal{B}^{(\alpha+2)}+M \delta(x-\xi)$.

In the same way we have discussed before, we can see that in this situation the Hermite case is not possible with $a_{1} \neq b_{1}$.

2. When $\operatorname{deg} \tilde{\beta}=3$ and $\operatorname{deg} \varphi_{2} \leq 2$, under the conditions described in Theorems $4.3-4.7, \mathcal{V}$ is a classical linear functional. If we assume $\mathcal{V}=\mathcal{J}^{(\alpha, \beta)}$ is associated with the Jacobi measure, then we have the pairs

$$
\begin{aligned}
d \mu_{0} & =(1-x)^{\alpha}(1+x)^{\beta} d x, \\
\left(J_{1,2}\right) \quad d \mu_{1} & =\frac{x-\xi_{0}}{x-\xi_{1}}(1-x)^{\alpha+1}(1+x)^{\beta+1} d x+M \delta\left(x-\xi_{1}\right),
\end{aligned}
$$

with $\alpha, \beta>-1$ and $\left|\xi_{0}\right|,\left|\xi_{1}\right| \geq 1$, as well as

$$
\left(J_{1,3}\right) \quad d \mu_{0}=(1-x)^{\alpha}(1+x)^{\beta} d x, \quad d \mu_{1}=(1-x)^{\alpha+1}(1+x)^{\beta+1} d x+M \delta(x-\xi),
$$

with $\alpha, \beta>-1$ and $|\xi|<1$.

If we assume $\mathcal{V}=\mathcal{L}^{(\alpha)}$ is associated with the Laguerre measure, then

$\left(L_{1,2}\right) \quad d \mu_{0}=x^{\alpha} e^{-x} d x, \quad d \mu_{1}=\frac{x-\xi_{0}}{x-\xi_{1}} x^{\alpha+1} e^{-x} d x+M \delta\left(x-\xi_{1}\right)$,

with $\alpha>-1$ and $\xi_{0}, \xi_{1} \leq 0$, and

$\left(L_{1,3}\right) \quad d \mu_{0}=x^{\alpha} e^{-x} d x, \quad d \mu_{1}=x^{\alpha+1} e^{-x} d x+M \delta(x-\xi)$,

with $\alpha>-1$ and $\xi>0$. 
In the above cases, the linear functional $\mathcal{V}$ satisfies the conditions described in [1]. For example, in $\left(J_{1,2}\right)$ we have $\left(x-\xi_{1}\right) \mathcal{U}=\left(x-\xi_{0}\right) \mathcal{J}^{(\alpha+1, \beta+1)}$. Then, the results shown in [1] give us a $2-2$ relation between the polynomials $P_{n}$ and the Jacobi polynomials $P_{n}^{(\alpha+1, \beta+1)}$. Taking into account that $R_{n}=P_{n}^{(\alpha, \beta)}$ as well as the differentiation formula for the Jacobi polynomials, $R_{n}^{\prime}=n P_{n-1}^{(\alpha+1, \beta+1)}$, then it is easy to prove that the pair $\left(J_{1,2}\right)$ satisfies a relation of the type (1.8). In a similar way, we can check this relation for $\left(J_{1,3}\right),\left(L_{1,2}\right)$, and $\left(L_{1,3}\right)$. Furthermore, cases $\left(J_{1,2}\right)$ and $\left(L_{1,2}\right)$ appear in the examples given by Sri Ranga et al. in [3].

Finally, if $\mathcal{V}=\mathcal{B}^{(\alpha)}$ is the Bessel linear functional, then we have

$\left(B_{1,2}\right) \quad \mathcal{U}=\left(x-\xi_{1}\right)^{-1}\left(x-\xi_{0}\right) \mathcal{B}^{(\alpha+2)}+M \delta\left(x-\xi_{1}\right)$.

We conclude this section with the cases when the linear functional $\mathcal{V}$ is semiclassical of class 1 , and we will describe the different pairs according to the classification given by $\mathrm{S}$. Belmehdi in [2].

3. If $\tilde{\beta}(x)=\left(x^{2}-1\right)(x-\lambda)$, then for the measures $\mu_{0}$ and $\mu_{1}$ associated with $\mathcal{V}$ and $\mathcal{U}$, respectively, we have

$$
\begin{aligned}
& d \mu_{0}=(1-x)^{\alpha}(1+x)^{\beta}|x-\lambda|^{\gamma} d x \\
& d \mu_{1}=\frac{1}{x-\xi}(1-x)^{\alpha+1}(1+x)^{\beta+1}|x-\lambda|^{\gamma+1} d x+M \delta(x-\xi),
\end{aligned}
$$

with $\alpha, \beta, \gamma>-1,|\alpha|+|\beta|+|\gamma| \neq 0$, and $|\xi| \geq 1$. The support of these measures is the interval $[-1,1]$.

4. If $\tilde{\beta}(x)=x^{2}(x-1)$, then we have the pair of linear functionals supported on the interval $[0,1]$ associated with the measures

$$
\begin{aligned}
& d \mu_{0}=(1-x)^{\alpha} x^{\beta} e^{\frac{-\gamma}{x}} d x, \\
& d \mu_{1}=\frac{1}{x-\xi}(1-x)^{\alpha+1} x^{\beta+2} e^{\frac{-\gamma}{x}} d x+M \delta(x-\xi),
\end{aligned}
$$

with $\alpha, \beta>-1, \gamma>0$, and $\xi \notin(0,1)$.

5. If $\tilde{\beta}(x)=x^{3}$, then we have the pair

$$
d \mu_{0}=x^{\alpha} e^{\frac{\beta}{x}-\frac{2}{x^{2}}} d x, \quad d \mu_{1}=\frac{1}{x-\xi} x^{\alpha+3} e^{\frac{\beta}{x}-\frac{2}{x^{2}}} d x+M \delta(x-\xi) .
$$

This is the analog of Bessel linear functional in the classical case, and the support of the measures is not on the real line.

6 . If $\tilde{\beta}(x)=x^{2}-1$, we have

$$
\begin{aligned}
& d \mu_{0}=(1-x)^{\alpha}(1+x)^{\beta} e^{-\lambda x} d x, \\
& d \mu_{1}=\frac{1}{x-\xi}(1-x)^{\alpha+1}(1+x)^{\beta+1} e^{-\lambda x} d x+M \delta(x-\xi),
\end{aligned}
$$

with $\alpha, \beta>-1,|\xi| \geq 1$, and supported on the interval $[-1,1]$.

7. When $\tilde{\beta}(x)=x^{2}$, the measures are supported on the half line $[0,+\infty)$,

$\left(S_{1,5}\right) \quad d \mu_{0}=x^{\alpha} e^{-x+\frac{\beta}{x}} d x, \quad d \mu_{1}=\frac{1}{x-\xi} x^{\alpha+2} e^{-x+\frac{\beta}{x}} d x+M \delta(x-\xi)$,

with $\alpha>-1, \beta<0$, and $\xi \leq 0$.

8. For $\tilde{\beta}(x)=x$ we have

$\left(S_{1,6}\right) \quad d \mu_{0}=x^{2 \mu} e^{-x^{2}-\lambda x} d x, \quad d \mu_{1}=\frac{1}{x-\xi} x^{2 \mu+1} e^{-x^{2}-\lambda x} d x+M \delta(x-\xi)$, 
supported on $[0,+\infty)$ with $\mu>-1 / 2$ and $\xi \leq 0$.

9. Finally, if $\tilde{\beta}(x)=1$, then we have the pair of complex measures

$$
\begin{aligned}
& d \mu_{0}=e^{\frac{i x^{3}}{3}-x^{2}+i(\alpha-1) x} d x, \\
& d \mu_{1}=\frac{1}{x-\xi_{0}} e^{\frac{i x^{3}}{3}-x^{2}+i(\alpha-1) x} d x+M \delta\left(x-\xi_{0}\right) .
\end{aligned}
$$

supported on the whole real line $\mathbb{R}$, with $\xi_{0} \notin \mathbb{R}$.

In order to check that all these pairs of linear functionals satisfy (1.8), we just have to take into account that the linear functional $\mathcal{V}$ is semiclassical of class 1 . The key property is shown in the next lemma.

LEMMA 4.9. Let $\mathcal{V}$ be a semiclassical quasi-definite linear functional of class 1 , satisfying $D[\phi \mathcal{V}]=\psi \mathcal{V}$, with $\operatorname{deg} \phi \leq 3$ and $\operatorname{deg} \psi \leq 2$, and let $\left\{R_{n}\right\}$ be the corresponding sequence of monic orthogonal polynomials. Assume that the linear functional $\mathcal{V}_{1}=\phi \mathcal{V}$ is quasi-definite, and denote by $\left\{S_{n}\right\}$ the corresponding sequence of monic orthogonal polynomials. Then, $R_{n+1}^{\prime}$ is quasi-orthogonal of order 1 with respect to $\mathcal{V}_{1}$, and

$$
\frac{R_{n+1}^{\prime}}{n+1}=S_{n}+\mu_{n} S_{n-1}
$$

with $\mu_{n} \neq 0$.

Proof. Taking into account that $\mathcal{V}$ is semiclassical of class 1 , it is well known [9] that we can represent the polynomial $\phi R_{n+1}^{\prime}$ as a linear combination of $R_{k}$ for $n-1 \leq k \leq n+3$, that is

$$
\phi R_{n+1}^{\prime}=c_{n, n+3} R_{n+3}+c_{n, n+2} R_{n+2}+c_{n, n+1} R_{n+1}+c_{n, n} R_{n}+c_{n, n-1} R_{n-1} .
$$

If we write $R_{n+1}^{\prime}=(n+1) S_{n}+\sum_{k=0}^{n-1} d_{n, k} S_{k}$, then it is easy to prove that $d_{n, k}=0$ for $k \leq n-2$, and then we deduce the result.

In our cases, the linear functional $\mathcal{V}$ satisfies $D[\tilde{\beta} \mathcal{V}]=-\varphi_{2} \mathcal{V}$, and the derivatives $R_{n+1}^{\prime}$ constitute a sequence of polynomials quasi-orthogonal with respect to the linear functional $\mathcal{V}_{1}=\tilde{\beta} \mathcal{V}$. Moreover, in all these cases $\mathcal{V}_{1}$ is again a quasi-definite semiclassical linear functional of class 1 . If we denote by $S_{n}$ the sequence of monic polynomials orthogonal with respect to $\mathcal{V}_{1}$, then we have

$$
\frac{R_{n+1}^{\prime}}{n+1}=S_{n}+\mu_{n} S_{n-1}
$$

with $\mu_{n} \neq 0$. Moreover, we can compute the coefficients $\mu_{n}$, taking into account $\mathcal{V}_{1}=\tilde{\beta} \mathcal{V}$. Indeed

$$
\begin{aligned}
\left\|S_{n-1}\right\|^{2} \mu_{n} & =\left\langle\mathcal{V}_{1}, \frac{R_{n+1}^{\prime}}{n+1} S_{n-1}\right\rangle=-\left\langle D[\tilde{\beta} \mathcal{V}], \frac{R_{n+1}}{n+1} S_{n-1}\right\rangle-\left\langle\tilde{\beta} \mathcal{V}, \frac{R_{n+1}}{n+1} S_{n-1}^{\prime}\right\rangle \\
& =\left\langle\mathcal{V}, \frac{R_{n+1}}{n+1}\left[\varphi_{2} S_{n-1}-\tilde{\beta} S_{n-1}^{\prime}\right]\right\rangle .
\end{aligned}
$$

Finally, from $(3.3)$ as well as $\tilde{\beta}=(x-c) \varphi_{2}-\varphi_{3}$, we obtain

$$
\mu_{n}=\left[\frac{3(n-1)}{n+1} \frac{a_{3}\left\|P_{2}\right\|^{2}}{b_{3}\left\|R_{3}\right\|^{2}}-\frac{2(n-2)}{n+1} \frac{a_{2}\left\|P_{1}\right\|^{2}}{b_{2}\left\|R_{2}\right\|^{2}}\right] \frac{\left\|R_{n+1}\right\|^{2}}{\left\|S_{n-1}\right\|^{2}} .
$$


On the other hand, the linear functional $\mathcal{V}_{1}$ is related to $\mathcal{U}$ by $(x-\xi) \mathcal{U}=\mathcal{V}_{1}$. Thus (see [9])

$$
P_{n}=S_{n}+\lambda_{n} S_{n-1},
$$

with $\lambda_{n} \neq 0$. More precisely, this coefficient is

$$
\lambda_{n}=\frac{\left\langle\mathcal{V}_{1}, P_{n} S_{n-1}\right\rangle}{\left\|S_{n-1}\right\|^{2}}=\frac{\left\langle\mathcal{U},(x-\xi) P_{n} S_{n-1}\right\rangle}{\left\|S_{n-1}\right\|^{2}}=\frac{\left\|P_{n}\right\|^{2}}{\left\|S_{n-1}\right\|^{2}},
$$

taking into account $\mathcal{V}_{1}=(x-\xi) \mathcal{U}$.

Since $(n+1) P_{n} \neq R_{n+1}^{\prime}$, then $\lambda_{n} \neq \mu_{n}$ for every $n \in \mathbb{N}$. Thus there exist $a_{n}$ and $b_{n}$ such that (1.8) holds. In fact,

$$
a_{n}=\frac{\left(\lambda_{n}-\mu_{n}\right) \mu_{n-1}}{\lambda_{n-1}-\mu_{n-1}}, \quad b_{n}=\frac{\left(\lambda_{n}-\mu_{n}\right) \lambda_{n-1}}{\lambda_{n-1}-\mu_{n-1}} .
$$

Finally, next table shows a list of all the possible cases of pairs of linear functionals satisfying (3.1), when $\mathcal{V}$ is a semiclassical linear functional of class at most 1 ,

\begin{tabular}{|l|l|l|}
\hline$\left(J_{1,1}\right)$ & $\begin{array}{l}\alpha, \beta>-1 \\
d \mu_{0}=(1-x)^{\alpha}(1+x)^{\beta} d x\end{array}$ & {$[-1,1]$} \\
$d \mu_{1}=\frac{1}{x-\xi}(1-x)^{\alpha+1}(1+x)^{\beta+1} d x+M \delta(x-\xi)$ & $|\xi| \geq 1$ & \\
\hline$\left(J_{1,2}\right)$ & $\begin{array}{l}\alpha, \beta>-1 \\
\left|\xi_{0}\right|,\left|\xi_{1}\right| \geq 1\end{array}$ & {$[-1,1]$} \\
$d \mu_{0}=(1-x)^{\alpha}(1+x)^{\beta} d x$ & & \\
$d \mu_{1}=\frac{x-\xi_{0}}{x-\xi_{1}}(1-x)^{\alpha+1}(1+x)^{\beta+1} d x+M \delta\left(x-\xi_{1}\right)$ & & {$[, \beta>-1$} \\
\hline$\left(J_{1,3}\right)$ & $|\xi|<1$ & {$[-1,1]$} \\
$d \mu_{0}=(1-x)^{\alpha}(1+x)^{\beta} d x$ & $\alpha>-1$ & {$[0,+\infty)$} \\
$d \mu_{1}=(1-x)^{\alpha+1}(1+x)^{\beta+1} d x+M \delta(x-\xi)$ & $\xi \leq 0$ & \\
\hline$\left(L_{1,1}\right)$ & $\begin{array}{l}\alpha>-1 \\
d \mu_{0}=x^{\alpha} e^{-x} d x,\end{array}$ & {$[0,+\infty)$} \\
$d \mu_{1}=\frac{1}{x-\xi} x^{\alpha+1} e^{-x} d x+M \delta(x-\xi)$ & $\xi_{0}, \xi_{1} \leq 0$ & \\
\hline$\left(L_{1,2}\right)$ & $\begin{array}{l}\alpha>-1 \\
d \mu_{0}=x^{\alpha} e^{-x} d x,\end{array}$ & {$[0,+\infty)$} \\
$d \mu_{1}=\frac{x-\xi_{0}}{x-\xi_{1}} x^{\alpha+1} e^{-x} d x+M \delta\left(x-\xi_{1}\right)$ & $\xi \notin 0$ & {$[0,1]$} \\
\hline$\left(L_{1,3}\right)$ & $|\xi| \geq 1$ & \\
$d \mu_{0}=x^{\alpha} e^{-x} d x$, & $\begin{array}{l}\alpha, \beta, \gamma>-1 \\
d \mu_{1}=x^{\alpha+1} e^{-x} d x+M \delta(x-\xi)\end{array}$ & {$[-1,1]$} \\
\hline$\left(S_{1,1}\right)$ & $|\alpha|+|\beta|+|\gamma| \neq 0$ & \\
$d \mu_{0}=(1-x)^{\alpha}(1+x)^{\beta}|x-\lambda|^{\gamma} d x$ & 1 \\
$d \mu_{1}=\frac{1}{x-\xi}(1-x)^{\alpha+1}(1+x)^{\beta+1}|x-\lambda|^{\gamma+1} d x$ & & \\
\hline$\left(S_{1,2}\right)$ & $+M \delta(x-\xi)$ & \\
$d \mu_{0}=(1-x)^{\alpha} x^{\beta} e^{\frac{-\gamma}{x}} d x$ & $1-x)^{\alpha+1} x^{\beta+2} e^{\frac{-\gamma}{x}} d x+M \delta(x-\xi)$ & \\
$d \mu_{1}=\frac{1}{x-\xi}(1-1)$ & \\
\hline
\end{tabular}




\begin{tabular}{|c|c|c|}
\hline $\begin{array}{l}\left(S_{1,3}\right) \\
d \mu_{0}=x^{\alpha} e^{\frac{\beta}{x}-\frac{2}{x^{2}}} d x \\
d \mu_{1}=\frac{1}{x-\xi} x^{\alpha+3} e^{\frac{\beta}{x}-\frac{2}{x^{2}}} d x+M \delta(x-\xi)\end{array}$ & & \\
\hline $\begin{array}{l}\left(S_{1,4}\right) \\
d \mu_{0}=(1-x)^{\alpha}(1+x)^{\beta} e^{-\lambda x} d x \\
d \mu_{1}=\frac{1}{x-\xi}(1-x)^{\alpha+1}(1+x)^{\beta+1} e^{-\lambda x} d x \\
\quad+M \delta(x-\xi)\end{array}$ & $\begin{array}{l}\alpha, \beta>-1 \\
|\xi| \geq 1\end{array}$ & {$[-1,1]$} \\
\hline $\begin{array}{l}\left(S_{1,5}\right) \\
d \mu_{0}=x^{\alpha} e^{-x+\frac{\beta}{x}} d x \\
d \mu_{1}=\frac{1}{x-\xi} x^{\alpha+2} e^{-x+\frac{\beta}{x}} d x+M \delta(x-\xi)\end{array}$ & $\begin{array}{l}\alpha>-1 \\
\beta<0 \\
\xi \leq 0\end{array}$ & {$[0,+\infty)$} \\
\hline $\begin{array}{l}\left(S_{1,6}\right) \\
d \mu_{0}=x^{2 \mu} e^{-x^{2}-\lambda x} d x \\
d \mu_{1}=\frac{1}{x-\xi} x^{2 \mu+1} e^{-x^{2}-\lambda x} d x+M \delta(x-\xi)\end{array}$ & $\begin{array}{l}\mu>-1 / 2 \\
\xi \leq 0\end{array}$ & {$[0,+\infty)$} \\
\hline $\begin{array}{l}\left(S_{1,7}\right) \\
d \mu_{0}=e^{\frac{i x^{3}}{3}-x^{2}+i(\alpha-1) x} d x \\
d \mu_{1}=\frac{1}{x-\xi_{0}} e^{\frac{i x^{3}}{3}-x^{2}+i(\alpha-1) x} d x+M \delta\left(x-\xi_{0}\right)\end{array}$ & $\xi_{0} \notin \mathbb{R}$ & $\mathbb{R}$ \\
\hline
\end{tabular}

\section{5. $\mathcal{U}$ is semiclassical of class at most 1 .}

5.1. Characterization of the pairs $(\mathcal{U}, \mathcal{V})$. In this section we analyze the case when the polynomial $\psi$ in Corollary 3.3 has two simple zeros. As in Section 4, we will see that with this assumption, if (1.8) holds, then the linear functional $\mathcal{U}$ must be semiclassical of class at most 1 , and moreover, the linear functional $\mathcal{V}$ can be represented as a rational modification of $\mathcal{U}$.

THEOREM 5.1. If the polynomial $\psi$ in (3.4) or (3.6) has two simple zeros, $\xi_{1} \neq \xi_{2}$, then $\mathcal{U}$ is a semiclassical linear functional of class at most 1 , that is, there exist polynomials $\tilde{\beta}, \varphi \in \mathbb{P}$, with $\operatorname{deg} \tilde{\beta} \leq 3$ and $\operatorname{deg} \varphi \leq 2$, such that

$$
D[\tilde{\beta} \mathcal{U}]=\varphi \mathcal{U}
$$

Moreover, the linear functional $\mathcal{V}$ is determined by

$$
\tilde{\beta} \mathcal{V}=(x-\xi) \mathcal{U},
$$

where $\xi \in\left\{\xi_{1}, \xi_{2}\right\}$.

To prove this theorem, we need several previous results.

Lemma 5.2. If $\xi$ is a simple zero of $\psi$ such that $\beta(\xi)=0$, then $\alpha(\xi)=0$ and $r_{1}(\xi) \neq 0$.

Proof. First, since $\xi$ is a simple zero of $\psi$, if we derive in (3.7) then we obtain $\psi^{\prime}(\xi)=r_{2}^{\prime}(\xi)+2 r_{1}(\xi)-r_{2}^{\prime}(\xi)=2 r_{1}(\xi) \neq 0$, thus $r_{1}(\xi) \neq 0$.

On the other hand, multiplying by $\beta$ in (3.8), from (3.4) and (3.5) we get $r_{1} \alpha=$ $-\beta-\varphi_{2} \psi$. Then, we evaluate in $\xi$ and obtain $r_{1}(\xi) \alpha(\xi)=0$. Since $r_{1}(\xi) \neq 0$, finally we deduce $\alpha(\xi)=0$. $\square$ 
LEMmA 5.3. If $\xi$ is such that $\psi(\xi)=0$ and $\beta(\xi) \neq 0$, then there exists a non-zero constant $C$ which is independent on $n$ such that

$$
r_{n}(\xi)+C r_{n}^{\prime}(\xi)=0
$$

for all $n \in \mathbb{N}$.

Proof. Multiplying by $\beta$ in (3.2) and using (3.4) and (3.5) we get

$$
\alpha r_{n}+\beta r_{n}^{\prime}=-\varphi_{n+1} \psi, n \geq 1 .
$$

Then, evaluation in $\xi$ yields $\alpha(\xi) r_{n}(\xi)+\beta(\xi) r_{n}^{\prime}(\xi)=0$. In particular, for $n=1$ we have $\alpha(\xi) r_{1}(\xi)+\beta(\xi)=0$. Since $\beta(\xi) \neq 0$, it must be $\alpha(\xi) \neq 0$. Thus the result follows taking $C=\beta(\xi) / \alpha(\xi)$.

Lemma 5.4. If there exist $\xi_{1}, \xi_{2}, C_{1}$, and $C_{2}$ such that

$$
r_{n}\left(\xi_{i}\right)+C_{i} r_{n}^{\prime}\left(\xi_{i}\right)=0, n \geq 1,
$$

for $i=1,2$, then $\xi_{1}=\xi_{2}$ and $C_{1}=C_{2}$.

Proof. We remember that $r_{n}$ can be expressed in terms of the polynomials $\left\{P_{n}\right\}$,

$$
r_{n}=P_{n}+\sum_{k=0}^{n-1} A_{n, k} P_{k},
$$

where the coefficients $A_{n, k}$ were given by condition $\left\langle r_{n} \mathcal{U}, T_{n+1}\right\rangle=0$, that is,

$$
\begin{aligned}
\left(a_{n+1}-b_{n+1}\right) & \left\langle\mathcal{U}, P_{n}^{2}\right\rangle-b_{n-1}\left(a_{n}-b_{n}\right) A_{n, n-1}\left\langle\mathcal{U}, P_{n-1}^{2}\right\rangle \\
+\ldots+(-1)^{n} b_{n+1} b_{n} \cdot \ldots \cdot b_{2}\left(a_{1}-b_{1}\right) A_{n, 0} & =0 .
\end{aligned}
$$

As we noticed after the proof of Lemma 3.1, we can choose the coefficients $A_{n, n-1}=\ldots=A_{n, 1}=0$, and

$$
A_{n}=A_{n, 0}=\frac{(-1)^{n+1}\left(a_{n+1}-b_{n+1}\right)\left\langle\mathcal{U}, P_{n}^{2}\right\rangle}{b_{n+1} b_{n} \cdot \ldots \cdot b_{2}\left(a_{1}-b_{1}\right)}
$$

so that we have $r_{n}=P_{n}+A_{n}$. Thus, (5.3) yields

$$
P_{n}\left(\xi_{i}\right)+C_{i} P_{n}^{\prime}\left(\xi_{i}\right)=-A_{n}, n \geq 1,
$$

for $i=1,2$. Or, equivalently,

$$
P_{n}\left(\xi_{1}\right)+C_{1} P_{n}^{\prime}\left(\xi_{1}\right)=P_{n}\left(\xi_{2}\right)+C_{2} P_{n}^{\prime}\left(\xi_{2}\right), n \geq 1,
$$

and the relation holds in a trivial way for $n=0$. Then, every polynomial $p \in \mathbb{P}$ satisfies

$$
p\left(\xi_{1}\right)+C_{1} p^{\prime}\left(\xi_{1}\right)=p\left(\xi_{2}\right)+C_{2} p^{\prime}\left(\xi_{2}\right) .
$$

In particular, for the polynomials $p(x)=\left(x-\xi_{2}\right)^{n}$ we get

$$
\left(\xi_{1}-\xi_{2}\right)^{n}+C_{1} n\left(\xi_{1}-\xi_{2}\right)^{n-1}=0, n \geq 2,
$$

from where we deduce $\xi_{1}=\xi_{2}$ and, finally, $C_{1}=C_{2}$. 
Now we are ready to prove the Theorem.

Proof of Theorem 5.1. Let $\xi_{1} \neq \xi_{2}$ be simple zeros of $\psi$. Then, from Lemma 5.3 and Lemma 5.4, one of them must be a zero of $\beta$. We can assume that $\psi\left(\xi_{1}\right)=$ $\beta\left(\xi_{1}\right)=0$. Then $\psi(x)=\left(x-\xi_{1}\right) \tilde{\psi}(x)$, with $\tilde{\psi}(x)=\left(x-\xi_{2}\right)$ and $\beta(x)=\left(x-\xi_{1}\right) \tilde{\beta}(x)$, with $\operatorname{deg} \tilde{\beta} \leq 3$. Moreover, from Lemma 5.2, $\xi_{1}$ must be a zero of $\alpha$. Then, $\alpha(x)=$ $\left(x-\xi_{1}\right) \tilde{\alpha}(x)$, with $\operatorname{deg} \tilde{\alpha} \leq 2$.

In such a situation, relations (3.4), (3.5), and (3.6) become

$$
\begin{gathered}
\tilde{\psi}(x) \mathcal{U}=\tilde{\beta}(x) \mathcal{V}+M \delta\left(x-\xi_{1}\right), \\
\tilde{\alpha}(x) \mathcal{U}=\tilde{\beta}(x) D \mathcal{U}+N \delta\left(x-\xi_{1}\right), \\
\tilde{\psi}(x) D \mathcal{U}=\tilde{\alpha}(x) \mathcal{V}+K \delta\left(x-\xi_{1}\right),
\end{gathered}
$$

and (5.2) becomes

$$
\tilde{\alpha} r_{n}+\tilde{\beta} r_{n}^{\prime}=-\varphi_{n+1} \tilde{\psi}, n \geq 1
$$

Then, using (3.2) and the previous relations we get

$$
\left(\tilde{\alpha} r_{n}+\tilde{\beta} r_{n}^{\prime}\right) \mathcal{V}=-\varphi_{n+1} \tilde{\psi} D\left[r_{n} \mathcal{U}\right]=\tilde{\psi}\left(r_{n}^{\prime} \mathcal{U}+r_{n} D \mathcal{U}\right)
$$

Thus we deduce $r_{n}(\tilde{\alpha} \mathcal{V}-\tilde{\psi} D \mathcal{U})=r_{n}^{\prime}(\tilde{\psi} \mathcal{U}-\tilde{\beta} \mathcal{V})$. Taking into account (5.4) and (5.6), we obtain

$$
K r_{n}\left(\xi_{1}\right)=-M r_{n}^{\prime}\left(\xi_{1}\right), n \geq 1
$$

Moreover, from Lemma 5.2 for $n=1$ we have $r_{1}\left(\xi_{1}\right) \neq 0$, as well as $r_{1}^{\prime}\left(\xi_{1}\right) \neq 0$. Then

$$
K=0 \Leftrightarrow M=0 .
$$

Now, for $\xi_{2}$ we have either $\tilde{\beta}\left(\xi_{2}\right) \neq 0$ or $\tilde{\beta}\left(\xi_{2}\right)=0$. First, we assume that $\tilde{\beta}\left(\xi_{2}\right) \neq 0$. From Lemma 5.3 there exists $C \neq 0$ such that $r_{n}\left(\xi_{2}\right)+C r_{n}^{\prime}\left(\xi_{2}\right)=0$ for $n \geq 1$. Thus, since $\xi_{1} \neq \xi_{2}$, by Lemma 5.4 we deduce $K=M=0$ in (5.8). On the other hand, if $\tilde{\beta}\left(\xi_{2}\right)=0$ then, in the same way that we have done the analysis for $\xi_{1}$, we get

$$
\tilde{K} r_{n}\left(\xi_{2}\right)=-\tilde{M} r_{n}^{\prime}\left(\xi_{2}\right), n \geq 1
$$

with $r_{1}\left(\xi_{2}\right) \neq 0$ and $r_{1}^{\prime}\left(\xi_{2}\right) \neq 0$. Thus, in this case we also obtain the condition

$$
\tilde{K}=0 \Leftrightarrow \tilde{M}=0 .
$$

As a consequence, Lemma 5.4 means that either (5.8) or (5.9) must be a trivial condition.

Then, we can assume that $K=M=0$ in (5.4) and (5.6). This proves the second assertion $\tilde{\beta}(x) \mathcal{V}=\left(x-\xi_{2}\right) \mathcal{U}$.

Furthermore, from (3.8) and (5.7) we deduce for $n=1$

$$
-\varphi_{2} \tilde{\beta} \mathcal{V}=\tilde{\beta} \mathcal{U}+r_{1} \tilde{\beta} D \mathcal{U}=-\left(\varphi_{2} \tilde{\psi}+r_{1} \tilde{\alpha}\right) \mathcal{U}+r_{1} \tilde{\beta} D \mathcal{U}
$$

and taking into account $(5.5)$ we get $N r_{1}\left(\xi_{1}\right)=0$. Finally, from Lemma $5.2, r_{1}\left(\xi_{1}\right) \neq$ 0 , thus $N=0$ in (5.5), and, as a consequence, the first statement follows with $\varphi=$ $\tilde{\alpha}+\tilde{\beta}^{\prime}$. 
In a similar way than in the case where the polynomial $\psi$ has a double zero, we need to describe the cases when relation (5.1) can be reduced to a classical one. That is, the polynomials $\tilde{\beta}$ and $\tilde{\alpha}$ have at least one common zero and moreover this zero can be simplified without addition of Dirac masses in the distributional Pearson equation.

We remember that the linear functional $\mathcal{U}$ satisfies $\tilde{\beta} D \mathcal{U}=\tilde{\alpha} \mathcal{U}$, and a priori the non-classical cases are those with either $\operatorname{deg} \tilde{\beta}=3$ and $\operatorname{deg} \tilde{\alpha} \leq 2$, or $\operatorname{deg} \tilde{\beta} \leq 2$ and $\operatorname{deg} \tilde{\alpha}=2$. If $\tilde{\beta}(x)=A x^{t}+$ l.d.t., then we define $\hat{\beta}(x)=A^{-1} a^{-t} \tilde{\beta}(a x+b)$ and $\hat{\alpha}(x)=A^{-1} a^{1-t} \tilde{\alpha}(a x+b)$. As a consequence, from Lemma 4.2, up to the linear change of variable $y \equiv a x+b$, the linear functional $\mathcal{U}$ satisfies

$$
\hat{\beta} D \mathcal{U}=\hat{\alpha} \mathcal{U} .
$$

Moreover, we denote $\hat{\alpha}(x)=\alpha_{2} x^{2}+\alpha_{1} x+\alpha_{0}$.

We classify the different cases according to the degree of the polynomial $\tilde{\beta}$.

JACOBI AND Bessel CASES: $\operatorname{deg} \tilde{\beta}=3$.

As in Section 4.1, taking into account Lemma 4.2, all the possible cases for $\tilde{\beta}$ can be reduced, up to a linear change of variables, to the canonical situations,

$$
\begin{aligned}
& \tilde{\beta}(x)=x^{3}, \\
& \tilde{\beta}(x)=x^{2}(x-1), \\
& \tilde{\beta}(x)=x(x-1)(x-\lambda) .
\end{aligned}
$$

Hence, without loss of generality, we may assume that $\tilde{\beta}$ has this expression.

Theorem 5.5. If $\tilde{\beta}(x)=x^{3}$, then $\mathcal{U}=\mathcal{B}^{(\alpha)}$ is the Bessel linear functional verifying the equation

$$
D\left[x^{2} \mathcal{U}\right]=\left[\left(\alpha_{2}+2\right) x+\alpha_{1}\right] \mathcal{U}
$$

if and only if $\alpha_{0}=0,\left(\alpha_{2}+2\right) \beta_{0}^{\mathcal{U}}+\alpha_{1}=0$ and the admissibility conditions $\alpha_{1}=2$ and $-\alpha_{2} \notin \mathbb{N}$ hold. Moreover, the linear functional $\mathcal{V}$ is given by

$$
\mathcal{V}=x^{-3}(x-\xi) \mathcal{B}^{(\alpha)}+M_{0} \delta(x)+M_{1} \delta^{\prime}(x)+M_{2} \delta^{\prime \prime}(x) .
$$

TheOREM 5.6. If $\tilde{\beta}$ has a double zero, then $\hat{\beta}(x)=x^{2}(x-1)$. The following two cases can appear.

i) $\mathcal{U}$ is the classical Jacobi linear functional satisfying

$$
D[x(x-1) \mathcal{U}]=\left[\left(\alpha_{2}+2\right) x+\alpha_{1}-1\right] \mathcal{U}
$$

if and only if $\alpha_{0}=0,\left(\alpha_{2}+2\right) \beta_{0}^{\mathcal{U}}+\alpha_{1}-1=0$, and the admissibility conditions $\alpha_{2}+\alpha_{1}+2 \notin \mathbb{N}, 2-\alpha_{1} \notin \mathbb{N}$, and $\alpha_{2}+4 \notin \mathbb{N}$ hold. Moreover, if $\mathcal{U}=\mathcal{J}^{(\alpha+1, \beta+2)}$ is the Jacobi linear functional associated with the measure $d \mu_{1}=(1-x)^{\alpha+1}(1+x)^{\beta+2} d x$, then the linear functional $\mathcal{V}$ verifies

$$
\mathcal{V}=(x-\xi) \mathcal{J}^{(\alpha, \beta)} .
$$

ii) $\mathcal{U}=\mathcal{B}^{(\alpha)}$ is the classical Bessel linear functional satisfying

$$
D\left[x^{2} \mathcal{U}\right]=\left[\left(\alpha_{2}+2\right) x-\alpha_{0}\right] \mathcal{U}
$$


if and only if $\alpha_{2}+\alpha_{1}+\alpha_{0}=0,\left(\alpha_{2}+2\right) \beta_{0}^{\mathcal{U}}-\alpha_{0}=0$, and the admissibility conditions $\alpha_{0}=-2$, and $-\alpha_{2} \notin \mathbb{N}$ hold. Moreover, the linear functional $\mathcal{V}$ is given by

$$
\mathcal{V}=x^{-2}(x-1)^{-1}(x-\xi) \mathcal{B}^{(\alpha)}+M_{0} \delta(x)+M_{1} \delta^{\prime}(x)+N \delta(x-1) .
$$

TheOREM 5.7. If $\tilde{\beta}(x)=x(x-1)(x-\lambda)$, then the following three cases can appear.

i) $\mathcal{U}$ is the classical Jacobi linear functional satisfying

$$
D[(x-1)(x-\lambda) \mathcal{U}]=\left[\left(\alpha_{2}+2\right) x+\alpha_{1}-\lambda-1\right] \mathcal{U}
$$

if and only if $\alpha_{0}=0,\left(\alpha_{2}+2\right) \beta_{0}^{\mathcal{U}}+\alpha_{1}-\lambda-1=0$, and the admissibility conditions $\left(\alpha_{1}+\alpha_{2} \lambda+2(\lambda-3)\right) /(\lambda-1) \notin \mathbb{N},\left(2(\lambda+1)-\alpha_{1}-\alpha_{2}\right) /(\lambda-1) \notin \mathbb{N}$ and $\alpha_{2}+4 \notin \mathbb{N}$ hold.

ii) $\mathcal{U}$ is the classical Jacobi linear functional satisfying

$$
D[x(x-\lambda) \mathcal{U}]=\left[\left(\alpha_{2}+2\right) x-\alpha_{0}-\lambda\right] \mathcal{U}
$$

if and only if $\alpha_{2}+\alpha_{1}+\alpha_{0}=0,\left(\alpha_{2}+2\right) \beta_{0}^{\mathcal{U}}-\alpha_{0}-\lambda=0$, and the admissibility conditions $2-\alpha_{0} / \lambda+\alpha_{2} \notin \mathbb{N}, \alpha_{0} / \lambda+2 \notin \mathbb{N}$ and $\alpha_{2}+4 \notin \mathbb{N}$ hold.

iii) $\mathcal{U}$ is the classical Jacobi linear functional satisfying

$$
D[x(x-1) \mathcal{U}]=\left[\left(\alpha_{2}+2\right) x-\alpha_{0} / \lambda-1\right] \mathcal{U}
$$

if and only if $\alpha_{2} \lambda^{2}+\alpha_{1} \lambda+\alpha_{0}=0,\left(\alpha_{2}+2\right) \beta_{0}^{\mathcal{U}}-\alpha_{0} / \lambda-1=0$, and the admissibility conditions $\alpha_{2}-\alpha_{0} / \lambda+2 \notin \mathbb{N}, \alpha_{0} / \lambda+2 \notin \mathbb{N}$, and $\alpha_{2}+4 \notin \mathbb{N}$ hold.

Moreover, if $\mathcal{U}=\mathcal{J}^{(\alpha+1, \beta+1)}$ is the Jacobi linear functional associated with the measure $d \mu_{1}=(1-x)^{\alpha+1}(1+x)^{\beta+1} d x$, then the linear functional $\mathcal{V}$ satisfies

$$
\mathcal{V}=\left(x-\xi_{1}\right)^{-1}\left(x-\xi_{0}\right) \mathcal{J}^{(\alpha, \beta)}+M \delta\left(x-\xi_{1}\right),
$$

with $\left|\xi_{0}\right|,\left|\xi_{1}\right| \geq 1$ or $\xi_{0}=\xi_{1}$ and $\left|\xi_{0}\right|<1$.

LAGUERRE CASES: $\operatorname{deg} \tilde{\beta}=2$.

In this case, by a linear change of variables we can make

$$
\begin{aligned}
& \tilde{\beta}(x)=x^{2}, \\
& \tilde{\beta}(x)=x(x-1) .
\end{aligned}
$$

Then, we may assume that $\tilde{\beta}$ has this expression without loss of generality.

TheOREm 5.8. If $\tilde{\beta}(x)=x^{2}$, then $\mathcal{U}$ is the classical Laguerre linear functional satisfying

$$
D[x \mathcal{U}]=\left[\alpha_{2} x+\alpha_{1}+1\right] \mathcal{U}
$$

if and only if $\alpha_{0}=0, \alpha_{2} \beta_{0}^{\mathcal{U}}+\alpha_{1}+1$, and the admissibility conditions $\alpha_{2}=-1$ and $-\alpha_{1} \notin \mathbb{N}$ hold. Moreover, if $\mathcal{U}=\mathcal{L}^{(\alpha+2)}$ is the Laguerre lineal functional associated with the measure $d \mu_{1}=x^{\alpha+2} e^{-x} d x$, then the linear functional $\mathcal{V}$ satisfies

$$
\mathcal{V}=(x-\xi) \mathcal{L}^{(\alpha)}+M \delta(x) .
$$


THEOREM 5.9. If $\tilde{\beta}(x)=x(x-1)$ thus, the following two situations can appear: i) $\mathcal{U}$ is the translated of the classical Laguerre linear functional satisfying

$$
D[(x-1) \mathcal{U}]=\left[\alpha_{2} x+\alpha_{1}+1\right] \mathcal{U}
$$

if and only if $\alpha_{0}=0, \alpha_{2} \beta_{0}^{\mathcal{U}}+\alpha_{1}+1=0$, and the admissibility conditions $\alpha_{2}=-1$ and $1-\alpha_{1} \notin \mathbb{N}$ hold.

ii) $\mathcal{U}$ is the classical Laguerre linear functional satisfying

$$
D[x \mathcal{U}]=\left[\alpha_{2} x-\alpha_{0}+1\right] \mathcal{U}
$$

if and only if $\alpha_{2}+\alpha_{1}+\alpha_{0}=0, \alpha_{2} \beta_{0}^{\mathcal{U}}-\alpha_{0}+1$, and the admissibility conditions $\alpha_{2}=-1$ and $\alpha_{0} \notin \mathbb{N}$ hold.

Moreover, if $\mathcal{U}=\mathcal{L}^{(\alpha+1)}$ is the Laguerre lineal functional associated with the measure $d \mu_{1}=x^{\alpha+1} e^{-x} d x$, then

$$
\mathcal{V}=\left(x-\xi_{1}\right)^{-1}\left(x-\xi_{0}\right) \mathcal{L}^{(\alpha)}+M \delta\left(x-\xi_{1}\right),
$$

with $\xi_{0}, \xi_{1} \leq 0$ or $\xi_{0}=\xi_{1}>0$.

HERMITE CASES: $\operatorname{deg} \tilde{\beta}=1$.

THEOREM 5.10. If $\tilde{\beta}(x)=x$ then $\mathcal{U}$ is the classical Hermite linear functional satisfying

$$
D[\mathcal{U}]=\left[\alpha_{2} x+\alpha_{1}\right] \mathcal{U}
$$

if and only if $\alpha_{0}=0, \alpha_{2} \beta_{0}^{\mathcal{U}}+\alpha_{1}=0$, and the admissibility conditions $\alpha_{2}=-2$ and $\alpha_{1}=0$ hold .

Notice that, as in the previous case, the Hermite case can not happen. In fact, if $\mathcal{U}=\mathcal{H}$ is the Hermite linear functional associated with the measure $d \mu_{1}=e^{-x^{2}} d x$, from (4.2) we have an analog situation for the linear functional $\mathcal{V}$ to that in Theorem 4.8 , which is not possible.

5.2. Classification. We finish our study with the description of the linear functional $\mathcal{V}$ when $\mathcal{U}$ is semiclassical of class at most 1 .

From Theorem 5.1, we have the following classification for the pair of linear functionals $\mathcal{U}$ and $\mathcal{V}$ associated with the measures $d \mu_{1}$ and $d \mu_{0}$, respectively.

10. When $\operatorname{deg} \tilde{\beta} \leq 2$ and $\operatorname{deg} \varphi=1$, by (5.1) $\mathcal{U}$ is a classical linear functional.

In the Jacobi case, $\mathcal{U}=\mathcal{J}^{(\alpha+1, \beta+1)}$, Theorem 4.1 gives the pairs

$\left(J_{2,1}\right) \quad d \mu_{0}=(x-\xi)(1-x)^{\alpha}(1+x)^{\beta} d x, \quad d \mu_{1}=(1-x)^{\alpha+1}(1+x)^{\beta+1} d x$,

with $\alpha, \beta>-1,|\xi| \geq 1$ and

$\left(J_{2,2}\right) \quad d \mu_{0}=(1+x)^{\beta} d x+M \delta(x-1), \quad d \mu_{1}=(1+x)^{\beta+1} d x$,

with $\beta>-1$.

In the Laguerre case, $\mathcal{U}=\mathcal{L}^{(\alpha+1)}$, we obtain the pairs

$\left(L_{2,1}\right) \quad d \mu_{0}=(x-\xi) x^{\alpha} e^{-x} d x, \quad d \mu_{1}=x^{\alpha+1} e^{-x} d x$,

with $\alpha>-1, \xi \leq 0$, as well as

$\left(L_{2,2}\right) \quad d \mu_{0}=e^{-x} d x+M \delta(x), \quad d \mu_{1}=e^{-x} d x$.

All the previous cases are positive-definite coherent pairs described in [14], and satisfy (1.8) with $a_{n}=0$. 
Finally, when $\mathcal{U}=\mathcal{B}^{(\alpha)}$ is the Bessel linear functional, then $\mathcal{V}$ is defined by

$\left(B_{2,1}\right) \quad \mathcal{V}=x^{-2}\left(x-\xi_{0}\right) \mathcal{B}^{(\alpha)}+M_{0} \delta(x)+M_{1} \delta^{\prime}(x)+N \delta\left(x-\xi_{1}\right)$.

11. On the other hand, when either $\operatorname{deg} \tilde{\beta} \leq 2$ and $\operatorname{deg} \varphi=2$, or $\operatorname{deg} \tilde{\beta}=3$ and $\operatorname{deg} \varphi \leq 2$ and $\mathcal{U}$ is a classical linear functional under the assumptions from Theorem 5.5 to Theorem 5.9, the classification of the pairs $\mathcal{U}$ and $\mathcal{V}$ is as follows.

If $\mathcal{U}=\mathcal{J}^{(\alpha+1, \beta+1)}$ is the Jacobi linear functional, then we get the pairs

$\begin{aligned}\left(J_{2,3}\right) \quad d \mu_{0} & =\frac{x-\xi_{0}}{x-\xi_{1}}(1-x)^{\alpha}(1+x)^{\beta} d x+M \delta\left(x-\xi_{1}\right), \\ d \mu_{1} & =(1-x)^{\alpha+1}(1+x)^{\beta+1} d x,\end{aligned}$

with $\alpha, \beta>-1$ and either $\left|\xi_{0}\right|,\left|\xi_{1}\right| \geq 1$ or $\xi_{0}=\xi_{1}$ if $\left|\xi_{1}\right|<1$, and finally

$\left(J_{2,4}\right) \quad d \mu_{0}=\frac{1}{x-\xi}(1+x)^{\beta} d x+M \delta(x-\xi)+N \delta(x-1), \quad d \mu_{1}=(1+x)^{\beta+1} d x$,

with $\beta>-1$ and $|\xi| \geq 1$.

In case $\left(J_{2,3}\right)$, the linear functional $\mathcal{V}$ satisfies the conditions described in [1], that is, $\left(x-\xi_{1}\right) \mathcal{V}=\left(x-\xi_{0}\right) \mathcal{J}^{(\alpha, \beta)}$. Then, as the authors show in [1], we have a $2-2$ relation for $R_{n}$ and the Jacobi polynomials $P_{n}^{(\alpha, \beta)}$, from where we obtain directly that this pair verifies (1.8).

To conclude with the Jacobi cases, we will analyze the pair $\left(J_{2,4}\right)$. We have that $(x-1)(x-\xi) \mathcal{V}=\mathcal{J}^{(1, \beta)}$. Thus, (see [9]) we can express $R_{n}$ as a linear combination of Jacobi polynomials $P_{n}^{(1, \beta)}, P_{n-1}^{(1, \beta)}$, and $P_{n-2}^{(1, \beta)}$, and as a consequence, the derivatives $R_{n+1}^{\prime}$ can be given as a linear combination of $P_{n}^{(2, \beta+1)}, P_{n-1}^{(2, \beta+1)}$, and $P_{n-2}^{(2, \beta+1)}$,

$$
\frac{R_{n+1}^{\prime}}{n+1}=P_{n}^{(2, \beta+1)}+c_{n} P_{n-1}^{(2, \beta+1)}+\hat{c}_{n} P_{n-2}^{(2, \beta+1)}, \quad n \geq 2,
$$

with $\hat{c}_{n} \neq 0$. On the other hand we have that the linear functional $\mathcal{U}$ verifies $(x-$ $1)^{2} \mathcal{U}=\mathcal{J}^{(2, \beta+1)}$. Then (see [9]) $P_{n}$ can also be represented as a linear combination of the Jacobi polynomials

$$
P_{n}=P_{n}^{(2, \beta+1)}+d_{n} P_{n-1}^{(2, \beta+1)}+\hat{d}_{n} P_{n-2}^{(2, \beta+1)}, \quad n \geq 2,
$$

with $\hat{d}_{n} \neq 0$. Then, it is easy to deduce that there exist $a_{n}$ and $b_{n} \neq 0$ such that (1.8) holds if and only if the coefficients verify the relations $c_{n}+b_{n}=d_{n}+a_{n}$, $\hat{c}_{n}+b_{n} c_{n-1}=\hat{d}_{n}+a_{n} d_{n-1}$, and $b_{n} \hat{c}_{n-1}=a_{n} \hat{d}_{n-1}$. Then, taking into account $\hat{c}_{n}=$ $[(n+1)(n-1)]^{-1}\left\|R_{n+1}\right\|^{2}\left\|P_{n-1}^{(1, \beta)}\right\|^{-2}$ and $\hat{d}_{n}=\left\|P_{n}\right\|^{2}\left\|P_{n-1}^{(2, \beta+1)}\right\|^{-2}$, two compatibility conditions are needed,

$$
\begin{gathered}
\frac{\left\|P_{n}\right\|^{2}}{\left\|P_{n-1}^{(2, \beta+1)}\right\|^{2}} \neq \frac{1}{(n+1)(n-1)} \frac{\left\|R_{n+1}\right\|^{2}}{\left\|P_{n-1}^{(1, \beta)}\right\|^{2}}, \quad n \geq 2, \\
\left(d_{n}-c_{n}\right)\left(\hat{d}_{n-1} c_{n-1}-\hat{c}_{n-1} d_{n-1}\right)=\left(\hat{d}_{n}-\hat{c}_{n}\right)\left(\hat{d}_{n-1}-\hat{c}_{n-1}\right), \quad n \geq 2 .
\end{gathered}
$$

In such a case, the coefficients $a_{n}$ and $b_{n}$ are given in terms of $c_{n}$ and $d_{n}$ by

$$
\left(n(n-2) \frac{\left\|P_{n-1}\right\|^{2}}{\left\|R_{n}\right\|^{2}}-1\right) b_{n}=d_{n}-c_{n}, \quad a_{n}=n(n-2) \frac{\left\|P_{n-1}\right\|^{2}}{\left\|R_{n}\right\|^{2}} b_{n} .
$$

In the Laguerre case, $\mathcal{U}=\mathcal{L}^{(\alpha+1)}$, we have the pairs 
$\left(L_{2,3}\right) \quad d \mu_{0}=\frac{x-\xi_{0}}{x-\xi_{1}} x^{\alpha} e^{-x} d x+M \delta\left(x-\xi_{1}\right), \quad d \mu_{1}=x^{\alpha+1} e^{-x} d x$,

with $\alpha>-1$ and either $\xi_{0}, \xi_{1} \leq 0$ or $\xi_{0}=\xi_{1}>0$, as well as

$\left(L_{2,4}\right) \quad d \mu_{0}=\frac{1}{x-\xi} e^{-x} d x+M \delta(x-\xi)+N \delta(x), \quad d \mu_{1}=e^{-x} d x$,

with $\xi<0$.

Condition (1.8) for the pair $\left(L_{2,3}\right)$ follows in a similar way that in case $\left(J_{2,3}\right)$, because the linear functional $\mathcal{V}$ satisfies the conditions studied in [1].

The case $\left(L_{2,4}\right)$ can be analyzed in a similar way to $\left(J_{2,4}\right)$. Here, $\mathcal{U}$ and $\mathcal{V}$ are related with the Laguerre linear functional by $x(x-\xi) \mathcal{V}=\mathcal{L}^{(1)}$ and $x^{2} \mathcal{U}=\mathcal{L}^{(2)}$. Then, taking into account that $L_{n+1}^{(1)^{\prime}}=(n+1) L_{n}^{(2)}$ as well as the results in [9], we deduce that both $R_{n+1}^{\prime}$ and $P_{n}$ can be expressed as a linear combination of the Laguerre polynomials $L_{n}^{(2)}, L_{n-1}^{(2)}$, and $L_{n-2}^{(2)}$,

$$
\frac{R_{n+1}^{\prime}}{n+1}=L_{n}^{(2)}+c_{n} L_{n-1}^{(2)}+\hat{c}_{n} L_{n-2}^{(2)}, \quad n \geq 2,
$$

with $\hat{c}_{n} \neq 0$, and

$$
P_{n}=L_{n}^{(2)}+d_{n} L_{n-1}^{(2)}+\hat{d}_{n} L_{n-2}^{(2)}, \quad n \geq 2,
$$

with $\hat{d}_{n} \neq 0$. Then, as in case $\left(J_{2,4}\right),(1.8)$ holds if and only if the compatibility conditions (5.10) and (5.11) hold. Moreover, coefficients $a_{n}$ and $b_{n}$ are determined from $c_{n}$ and $d_{n}$ by (5.12).

The last classical case, when $\mathcal{U}=\mathcal{B}^{(\alpha)}$ is the Bessel linear functional, gives us the pair

$\left(B_{2,2}\right) \quad \mathcal{V}=x^{-2}\left(x-\xi_{1}\right)^{-1}\left(x-\xi_{0}\right) \mathcal{B}^{(\alpha)}+M_{0} \delta(x)+M_{1} \delta^{\prime}(x)+N \delta\left(x-\xi_{1}\right)$.

Finally, when $\mathcal{U}$ is a semiclassical linear functional of class 1 , we describe the pairs of linear functionals using the classification in [2].

12. If $\tilde{\beta}$ has three simple zeros, we deduce the pair of linear functionals associated with the following measures supported on the interval $[-1,1]$,

$$
\begin{aligned}
& d \mu_{0}=(x-\xi)(1-x)^{\alpha}(1+x)^{\beta}(x-c)^{\gamma} d x, \\
& d \mu_{1}=(1-x)^{\alpha+1}(1+x)^{\beta+1}(x-c)^{\gamma+1} d x,
\end{aligned}
$$

with $\alpha, \beta, \gamma>-1,|\alpha|+|\beta|+|\gamma| \neq 0$, and $|c|>1$. Notice that, in case that $\gamma=0$ (and the analogous $\alpha=0$ or $\beta=0$ ), we recover the example studied by Sri Ranga et al. in [3].

13. If $\tilde{\beta}=x^{2}(x-1)$, we have three different pairs supported on the interval $[0,1]$,

$\left(S_{2,2}\right) \quad d \mu_{0}=(x-\xi)(1-x)^{\alpha} x^{\beta} e^{-\gamma / x} d x, \quad d \mu_{1}=(1-x)^{\alpha+1} x^{\beta+2} e^{-\gamma / x} d x$,

with $\alpha, \beta>-1,|\alpha|+|\beta| \neq 0$, and $\gamma>0$, as well as

$\left(S_{2,3}\right) \quad d \mu_{0}=x^{\beta} e^{-\gamma / x} d x+M \delta(x-1), \quad d \mu_{1}=x^{\beta+2} e^{-\gamma / x} d x$,

with $\beta>-1, \beta \neq 0$, and $\gamma>0$, and

$\left(S_{2,4}\right) \quad d \mu_{0}=(1-x)^{\alpha} e^{-\gamma / x} d x+M \delta(x), \quad d \mu_{1}=(1-x)^{\alpha+1} x e^{-\gamma / x} d x$,

with $\alpha>-1, \alpha \neq 0$, and $\gamma>0$.

14. If $\tilde{\beta}$ has a zero of multiplicity 3 , then

$$
d \mu_{0}=(x-\xi) x^{\alpha} e^{\frac{\beta}{x}-\frac{2}{x^{2}}} d x, \quad d \mu_{1}=x^{\alpha+3} e^{\frac{\beta}{x}-\frac{2}{x^{2}}} d x .
$$


This is the analogous of Bessel in the classical case, and its support is not on the real line.

15. If $\tilde{\beta}=x^{2}-1$, then we deduce the pairs of linear functionals associated with the following measures supported on the half line $[1,+\infty)$,

$$
\begin{aligned}
& d \mu_{0}=(x-\xi)(1-x)^{\alpha}(1+x)^{\beta} e^{-\lambda x} d x, \\
& d \mu_{1}=(1-x)^{\alpha+1}(1+x)^{\beta+1} e^{-\lambda x} d x,
\end{aligned}
$$

with $\alpha>-1,|\alpha|+|\beta| \neq 0, \lambda>0$, and $\xi \leq 1$. Notice that for $\beta=0$, we recover the example given by Sri Ranga et al. in [3], up to a linear transformation of the variable $x$.

$$
d \mu_{0}=\frac{x-\xi_{0}}{x-\xi_{1}}(1-x)^{\alpha} e^{-\lambda x} d x+M \delta\left(x-\xi_{1}\right), \quad d \mu_{1}=(1-x)^{\alpha+1} e^{-\lambda x} d x .
$$

with $\alpha>-1, \alpha \neq 0, \lambda>0$, and $\xi_{0}, \xi_{1} \leq 1$, and

$\left(S_{2,8}\right) \quad d \mu_{0}=(1+x)^{\beta} e^{-\lambda x} d x+M \delta(x-1), \quad d \mu_{1}=(1+x)^{\beta+1} e^{-\lambda x} d x$,

with $\beta \neq 0$ and $\lambda>0$.

16. If $\tilde{\beta}$ has a double zero, then the linear functionals are associated with measures supported on $[0,+\infty)$, and we deduce the pairs

$\left(S_{2,9}\right) \quad d \mu_{0}=(x-\xi) x^{\alpha} e^{-x+\frac{\beta}{x}} d x, \quad d \mu_{1}=x^{\alpha+2} e^{-x+\frac{\beta}{x}} d x$,

with $\beta<0$ and $\alpha>-1$ and $\xi \leq 0$, and

$\left(S_{2,10}\right) \quad d \mu_{0}=e^{-x+\frac{\beta}{x}} d x+M \delta(x), \quad d \mu_{1}=x e^{-x+\frac{\beta}{x}} d x$,

with $\beta<0$.

17. If $\tilde{\beta}$ is a polynomial of degree 1 , then we have the pair of linear functionals associated with measures supported on the half line $[0,+\infty)$,

$\left(S_{2,11}\right) \quad d \mu_{0}=(x-\xi) x^{2 \mu} e^{-x^{2}-\lambda x} d x, \quad d \mu_{1}=x^{2 \mu+1} e^{-x^{2}-\lambda x} d x$,

with $\mu>-1 / 2$ and $\xi \leq 0$, and

$\left(S_{2,12}\right) \quad d \mu_{0}=e^{-x^{2}-\lambda x} d x+M \delta(x), \quad d \mu_{1}=e^{-x^{2}-\lambda x} d x$.

18. Finally, when $\operatorname{deg} \tilde{\beta}=1$, we have two complex measures

$\left(S_{2,13}\right) \quad d \mu_{0}=(x-\xi) e^{i \frac{x^{3}}{3}-x^{2}+i(\alpha-1) x} d x, \quad d \mu_{1}=e^{i \frac{x^{3}}{3}-x^{2}+i(\alpha-1) x} d x$,

supported on the whole real line $\mathbb{R}$.

Notice that all the previous cases, but $\left(S_{2,3}\right)$ and $\left(S_{2,4}\right),\left(S_{2,7}\right)$ and $\left(S_{2,8}\right),\left(S_{2,10}\right)$, and $\left(S_{2,12}\right)$, respond to the same pattern. We will study the first case, $\left(S_{2,1}\right)$, to check that (1.8) holds.

We have that the linear functional $\mathcal{U}$ is semiclassical of class 1 verifying $D[\tilde{\beta} \mathcal{U}]=$ $\varphi \mathcal{U}$. If we consider the linear functional $\mathcal{U}_{1}$, associated with the measure

$$
d \hat{\mu}_{1}=(1-x)^{\alpha}(1+x)^{\beta}(x-c)^{\gamma} d x,
$$

then we have $\tilde{\beta} \mathcal{U}_{1}=\mathcal{U}$. Moreover, $\mathcal{U}_{1}$ is semiclassical of class 1 verifying $D\left[\tilde{\beta} \mathcal{U}_{1}\right]=\tau \mathcal{U}_{1}$. If we denote by $\left\{S_{n}\right\}$ the polynomials orthogonal with respect to $\mathcal{U}_{1}$ then, by Lemma 4.9 , the derivatives $S_{n+1}^{\prime}$ are quasi-orthogonal with respect to the linear functional $\mathcal{U}$. This means,

$$
\frac{S_{n+1}^{\prime}}{n+1}=P_{n}+c_{n} P_{n-1}
$$


with $c_{n} \neq 0$. On the other hand, the linear functional $\mathcal{V}$ is related with $\mathcal{U}_{1}$ by $\mathcal{V}=(x-\xi) \mathcal{U}_{1}$. Thus (see $\left.[9]\right)$

$$
S_{n}=R_{n}+d_{n} R_{n-1}
$$

Finally, both previous relations yield (1.8).

In the remaining cases, $\left(S_{2,3}\right)$ and $\left(S_{2,4}\right),\left(S_{2,7}\right)$, and $\left(S_{2,8}\right),\left(S_{2,10}\right)$, and $\left(S_{2,12}\right)$, relation (1.8) follows in the same way, similar to the case $\left(J_{2,4}\right)$. We will analyze here the case $\left(S_{2,3}\right)$. Let denote by $\mathcal{S}^{(\alpha, \beta)}$ the semiclassical linear functional associated with the measure $d \hat{\mu}=(1-x)^{\alpha} x^{\beta} e^{-\gamma / x} d x$, that verifies the Pearson differential equation $D\left[x^{2}(x-1) \mathcal{S}^{(\alpha, \beta)}\right]=\tau \mathcal{S}^{(\alpha, \beta)}$, and $\left\{S_{n}^{(\alpha, \beta)}\right\}$ the corresponding sequence of monic orthogonal polynomials. Then, the linear functional $\mathcal{V}$ is related with $\mathcal{S}^{(1, \beta)}$ by $(x-1) \mathcal{V}=\mathcal{S}^{(1, \beta)}$. Then (see [9]), $R_{n}$ can be represented as a linear combination of $S_{n}^{(1, \beta)}$ and $S_{n-1}^{(1, \beta)}$. Then, from Lemma 4.9 we have the derivatives $R_{n+1}^{\prime}$ in terms of $S_{n}^{(2, \beta+2)}, S_{n-1}^{(2, \beta+2)}$, and $S_{n-2}^{(2, \beta+2)}$. On the other hand, the linear functional $\mathcal{U}$ verifies $(x-1)^{2} \mathcal{U}=\mathcal{S}^{(2, \beta+2)}$. Thus, $P_{n}$ can also be expressed as a linear combination of $S_{n}^{(2, \beta+2)}, S_{n-1}^{(2, \beta+2)}$, and $S_{n-2}^{(2, \beta+2)}$. Then, proceeding in a similar way than in case $\left(J_{2,4}\right)$ we obtain necessary and sufficient conditions so that (1.8) holds.

Finally, next table shows a list of all the possible cases of pairs of linear functionals satisfying (3.1), when $\mathcal{U}$ is a semiclassical linear functional of class at most 1 .

\begin{tabular}{|c|c|c|}
\hline $\begin{array}{l}\left(J_{2,1}\right) \\
d \mu_{0}=(x-\xi)(1-x)^{\alpha}(1+x)^{\beta} d x \\
d \mu_{1}=(1-x)^{\alpha+1}(1+x)^{\beta+1} d x\end{array}$ & $\begin{array}{l}\alpha, \beta>-1 \\
|\xi| \geq 1\end{array}$ & {$[-1,1]$} \\
\hline $\begin{array}{l}\left(J_{2,2}\right) \\
d \mu_{0}=(1+x)^{\beta} d x+M \delta(x-1) \\
d \mu_{1}=(1+x)^{\beta+1} d x\end{array}$ & $\beta>-1$ & {$[-1,1]$} \\
\hline $\begin{array}{l}\left(J_{2,3}\right) \\
d \mu_{0}=\frac{x-\xi_{0}}{x-\xi_{1}}(1-x)^{\alpha}(1+x)^{\beta} d x+M \delta\left(x-\xi_{1}\right) \\
d \mu_{1}=(1-x)^{\alpha+1}(1+x)^{\beta+1} d x\end{array}$ & $\begin{array}{l}\alpha, \beta>-1 \\
\left|\xi_{0}\right|,\left|\xi_{1}\right| \geq 1 \\
\xi_{0}=\xi_{1} \text { if }\left|\xi_{1}\right|<1\end{array}$ & {$[-1,1]$} \\
\hline $\begin{array}{l}\left(J_{2,4}\right) \\
d \mu_{0}=\frac{1}{x-\xi}(1+x)^{\beta} d x+M \delta(x-\xi)+N \delta(x-1) \\
d \mu_{1}=(1+x)^{\beta+1} d x\end{array}$ & $\begin{array}{l}\beta>-1 \\
|\xi| \geq 1\end{array}$ & {$[-1,1]$} \\
\hline $\begin{array}{l}\left(L_{2,1}\right) \\
d \mu_{0}=(x-\xi) x^{\alpha} e^{-x} d x \\
d \mu_{1}=x^{\alpha+1} e^{-x} d x\end{array}$ & $\begin{array}{l}\alpha>-1 \\
\xi \leq 0\end{array}$ & {$[0,+\infty)$} \\
\hline $\begin{array}{l}\left(L_{2,2}\right) \\
d \mu_{0}=e^{-x} d x+M \delta(x) \\
d \mu_{1}=e^{-x} d x\end{array}$ & & {$[0,+\infty)$} \\
\hline $\begin{array}{l}\left(L_{2,3}\right) \\
d \mu_{0}=\frac{x-\xi_{0}}{x-\xi_{1}} x^{\alpha} e^{-x} d x+M \delta\left(x-\xi_{1}\right) \\
d \mu_{1}=x^{\alpha+1} e^{-x} d x\end{array}$ & $\begin{array}{l}\alpha>-1 \\
\xi_{0}, \xi_{1} \leq 0 \\
\xi_{0}=\xi_{1}>0\end{array}$ & {$[0,+\infty)$} \\
\hline
\end{tabular}




\begin{tabular}{|c|c|c|}
\hline $\begin{array}{l}\left(L_{2,4}\right) \\
d \mu_{0}=\frac{1}{x-\xi} e^{-x} d x+M \delta(x-\xi)+N \delta(x) \\
d \mu_{1}=e^{-x} d x\end{array}$ & $\xi<0$ & {$[0,+\infty)$} \\
\hline $\begin{array}{l}\left(S_{2,1}\right) \\
d \mu_{0}=(x-\xi)(1-x)^{\alpha}(1+x)^{\beta}(x-c)^{\gamma} d x \\
d \mu_{1}=(1-x)^{\alpha+1}(1+x)^{\beta+1}(x-c)^{\gamma+1} d x\end{array}$ & $\begin{array}{l}\alpha, \beta, \gamma>-1 \\
|\alpha|+|\beta|+|\gamma| \neq 0 \\
|c|>1\end{array}$ & {$[-1,1]$} \\
\hline $\begin{array}{l}\left(S_{2,2}\right) \\
d \mu_{0}=(x-\xi)(1-x)^{\alpha} x^{\beta} e^{-\gamma / x} d x \\
d \mu_{1}=(1-x)^{\alpha+1} x^{\beta+2} e^{-\gamma / x} d x\end{array}$ & $\begin{array}{l}\alpha, \beta>-1 \\
|\alpha|+|\beta| \neq 0 \\
\gamma>0\end{array}$ & {$[0,1]$} \\
\hline $\begin{array}{l}\left(S_{2,3}\right) \\
d \mu_{0}=x^{\beta} e^{-\gamma / x} d x+M \delta(x-1) \\
d \mu_{1}=x^{\beta+2} e^{-\gamma / x} d x\end{array}$ & $\begin{array}{l}\beta>-1 \\
\beta \neq 0 \\
\gamma>0\end{array}$ & {$[0,1]$} \\
\hline $\begin{array}{l}\left(S_{2,4}\right) \\
d \mu_{0}=(1-x)^{\alpha} e^{-\gamma / x} d x+M \delta(x) \\
d \mu_{1}=(1-x)^{\alpha+1} x e^{-\gamma / x} d x\end{array}$ & $\begin{array}{l}\alpha>-1 \\
\alpha \neq 0 \\
\gamma>0\end{array}$ & {$[0,1]$} \\
\hline $\begin{array}{l}\left(S_{2,5}\right) \\
d \mu_{0}=(x-\xi) x^{\alpha} e^{\frac{\beta}{x}-\frac{2}{x^{2}}} d x \\
d \mu_{1}=x^{\alpha+3} e^{\frac{\beta}{x}-\frac{2}{x^{2}}} d x\end{array}$ & & \\
\hline $\begin{array}{l}\left(S_{2,6}\right) \\
d \mu_{0}=(x-\xi)(1-x)^{\alpha}(1+x)^{\beta} e^{-\lambda x} d x \\
d \mu_{1}=(1-x)^{\alpha+1}(1+x)^{\beta+1} e^{-\lambda x} d x\end{array}$ & $\begin{array}{l}\alpha>-1 \\
|\alpha|+|\beta| \neq 0 \\
\lambda>0 \\
\xi \leq 1 \\
\end{array}$ & {$[1,+\infty)$} \\
\hline $\begin{array}{l}\left(S_{2,7}\right) \\
d \mu_{0}=\frac{x-\xi_{0}}{x-\xi_{1}}(1-x)^{\alpha} e^{-\lambda x} d x+M \delta\left(x-\xi_{1}\right) \\
d \mu_{1}=(1-x)^{\alpha+1} e^{-\lambda x} d x\end{array}$ & $\begin{array}{l}\alpha>-1, \alpha \neq 0 \\
\lambda>0 \\
\xi_{0}, \xi_{1} \leq 1\end{array}$ & {$[1,+\infty)$} \\
\hline $\begin{array}{l}\left(S_{2,8}\right) \\
d \mu_{0}=(1+x)^{\beta} e^{-\lambda x} d x+M \delta(x-1) \\
d \mu_{1}=(1+x)^{\beta+1} e^{-\lambda x} d x\end{array}$ & $\begin{array}{l}\beta \neq 0 \\
\lambda>0\end{array}$ & {$[1,+\infty)$} \\
\hline $\begin{array}{l}\left(S_{2,9}\right) \\
d \mu_{0}=(x-\xi) x^{\alpha} e^{-x+\frac{\beta}{x}} d x \\
d \mu_{1}=x^{\alpha+2} e^{-x+\frac{\beta}{x}} d x\end{array}$ & $\begin{array}{l}\beta<0, \alpha>-1 \\
\xi \leq 0\end{array}$ & {$[0,+\infty)$} \\
\hline $\begin{array}{l}\left(S_{2,10}\right) \\
d \mu_{0}=e^{-x+\frac{\beta}{x}} d x+M \delta(x) \\
d \mu_{1}=x e^{-x+\frac{\beta}{x}} d x\end{array}$ & $\beta<0$ & {$[0,+\infty)$} \\
\hline $\begin{array}{l}\left(S_{2,11}\right) \\
d \mu_{0}=(x-\xi) x^{2 \mu} e^{-x^{2}-\lambda x} d x \\
d \mu_{1}=x^{2 \mu+1} e^{-x^{2}-\lambda x} d x\end{array}$ & $\begin{array}{l}\mu>-1 / 2 \\
\xi \leq 0\end{array}$ & {$[0,+\infty)$} \\
\hline $\begin{array}{l}\left(S_{2,12}\right) \\
d \mu_{0}=e^{-x^{2}-\lambda x} d x+M \delta(x) \\
d \mu_{1}=e^{-x^{2}-\lambda x} d x\end{array}$ & & {$[0,+\infty)$} \\
\hline
\end{tabular}




\begin{tabular}{|l|l|l|}
\hline$\left(S_{2,13}\right)$ & & \\
$d \mu_{0}=(x-\xi) e^{i \frac{x^{3}}{3}-x^{2}+i(\alpha-1) x} d x$ & & $\mathbb{R}$ \\
$d \mu_{1}=e^{i \frac{x^{3}}{3}-x^{2}+i(\alpha-1) x} d x$ & & \\
\hline
\end{tabular}

Acknowledgments. The work of the authors has been supported by Dirección General de Investigación, Ministerio de Ciencia y Tecnología of Spain, under grant BFM 2003-06335-C03-02 and INTAS Research Network NeCCA INTAS 03-31-6637.

This paper was finished during a stay of the authors in the School of Mathematics of Georgia Institute of Technology. We thank Prof. J. S. Geronimo for the help and suggestions to improve the manuscript.

Finally, we thank the referees for their helpful comments and suggestions which have improved the presentation of the paper.

\section{REFERENCES}

[1] M. Alfaro, F. Marcellán, A. Peña, M. L. Rezola, On linearly related orthogonal polynomials and their functionals, J. Math. Anal. Appl., 287:1(2003), pp. 307-319.

[2] S. Belmend, On semi-classical linear functionals of class $s=1$. Classification and integral representations, Indag. Mathem. N. S., 3(1992), pp. 253-275.

[3] A. C. Berti, C. F. Bracciali, A. SRi Ranga, Orthogonal Polynomials associated with related measures and Sobolev Orthogonal Polynomials, Num. Algorithms. In press.

[4] A. C. Berti, A. Sri Ranga, Companion orthogonal polynomials: some applications, Appl. Numer. Math., 39(2001), pp. 127-149.

[5] T. S. Chinara, An introduction to orthogonal polynomials, Gordon and Breach, New York, 1978.

[6] A. Iserles, P. E. Koch, S. P. Nørsett, J. M. Sanz-Serna, On polynomials orthogonal with respect to certain Sobolev inner products, J. Approx. Theory, 65(1991), pp. 151-175.

[7] D. H. Kim, K. H. Kwon, F. Marcellán, G. J. Yoon, Sobolev orthogonality and coherent pairs of moment functionals: an inverse problem, Intern. Math. Journal, 9(2002), pp. 877-888.

[8] F. Marcellán, H. G. Meijer, T. E. PÉrez, M. A. Piñar, An asymptotic result for LaguerreSobolev orthogonal polynomials, J. Comp. Appl. Math., 87(1997), pp. 87-94.

[9] P. Maroni, Une théorie algébrique des polyômes orthogonaux: Application aux polynômes orthogonaux semi-classiques. In Orthogonal polynomials and their applications, C. Brezinski et al. Editors. IMACS Ann. Comput. Appl. Math., 9, Baltzer, Basel, 1991. pp. 95-130.

[10] P. Maroni, Semi-classical character and finite-type relations between polynomial sequences, Appl. Num. Math., 31(1999), pp. 295-330.

[11] P. Maroni, R. Sfaxi, Diagonal orthogonal polynomial sequences, Meth. Appl. of Anal., 7(2000), pp. 769-792.

[12] A. Martínez Finkelshtein, Asymptotic properties of Sobolev orthogonal polynomials, J. Comp. Appl. Math., 99(1998), pp. 491-510.

[13] A. Martínez Finkelshtein, J. J. Moreno Balcázar, T. E. Pérez, M. A. Piñar, Asymptotics of Sobolev orthogonal polynomials for coherent pairs of measures, J. Approx. Theory, 92(1998), pp. 280-293.

[14] H. G. MeiJer, Determination of all coherent pairs, J. Approx. Theory, 89(1997), pp. 321-343.

[15] H. G. Meijer, T. E. PÉrez, M. A. Piñar, Asymptotic of Sobolev orthogonal polynomials for coherent pairs of Laguerre type, J. Math. Anal. Appl., 245(2000), pp. 528-546. 